\title{
Brain Cancer Drug Discovery: Clinical Trials, Drug Classes, Targets, and Combinatorial Therapies ${ }^{\mathbb{S}}$
}

\author{
Aleksandr V. Sokolov, Samira A. Dostdar, Misty M. Attwood, Aleksandra A. Krasilnikova, Anastasia A. Ilina, Amina Sh. Nabieva, \\ Anna A. Lisitsyna, Vladimir N. Chubarev, Vadim V. Tarasov, and Helgi B. Schiöth \\ Department of Neuroscience, Functional Pharmacology, Uppsala University, Uppsala, Sweden (A.V.S., S.A.D., M.M.A., H.B.S.); and \\ Department of Pharmacology, Institute of Pharmacy (A.V.S., S.A.D., A.A.K., A.A.I., A.S.N., A.A.L., V.N.C., V.V.T.) and Institute of \\ Translational Medicine and Biotechnology (V.V.T., H.B.S.), I. M. Sechenov First Moscow State Medical University, Moscow, Russia
}

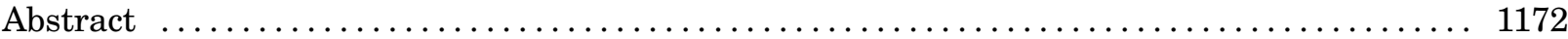

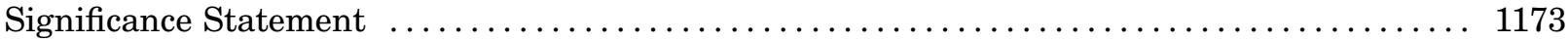

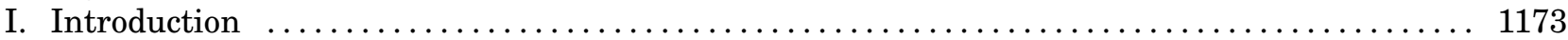

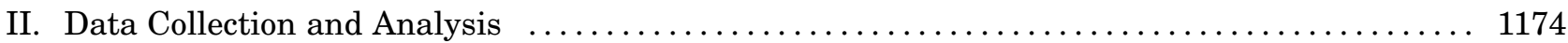

III. Overall Trends in Drugs, Drug Targets, and Biologic Therapies $\ldots \ldots \ldots \ldots \ldots \ldots \ldots \ldots \ldots 1175$

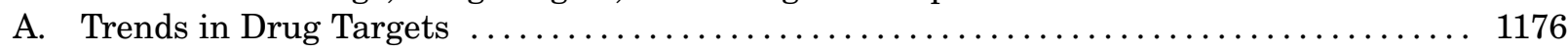

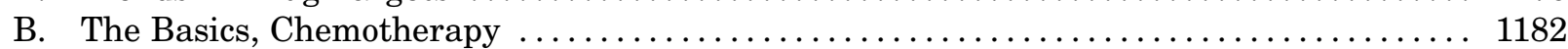

C. Gene Therapy Is Found in Six Classes $\ldots \ldots \ldots \ldots \ldots \ldots \ldots \ldots \ldots \ldots \ldots \ldots \ldots \ldots \ldots$

D. Checkpoint Inhibitors and Immune Modulators $\ldots \ldots \ldots \ldots \ldots \ldots \ldots \ldots \ldots \ldots \ldots$

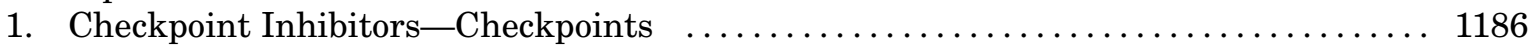

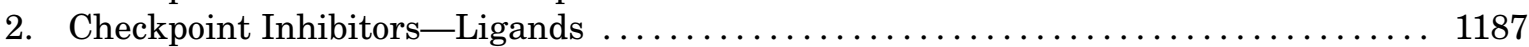

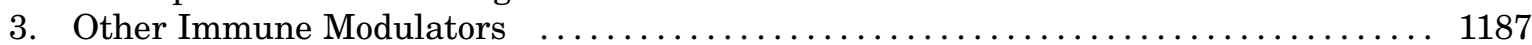

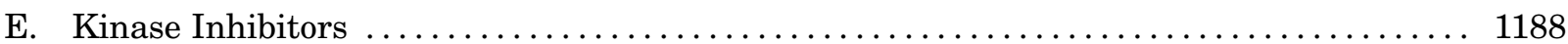

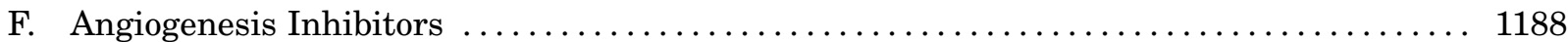

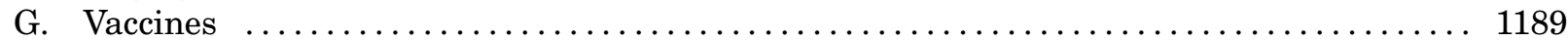

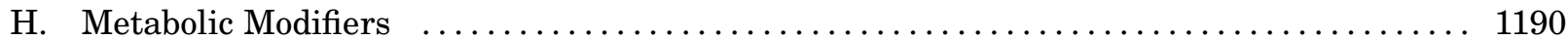

I. Transcription Modifiers: Tumor Targeting at a Different Level $\ldots \ldots \ldots \ldots \ldots \ldots \ldots \ldots \ldots 1191$

J. Oncolytic Viruses Are Still in Early Development $\ldots \ldots \ldots \ldots \ldots \ldots \ldots \ldots \ldots \ldots \ldots \ldots . \ldots \ldots 2$

K. PARP Inhibitors: Now beyond Breast Cancer . . . . . . . . . . . . . . . . . . . . . . . . . 1192

L. Radiopharmaceuticals: A Targeted Radiation Therapy $\ldots \ldots \ldots \ldots \ldots \ldots \ldots \ldots \ldots \ldots 1192$

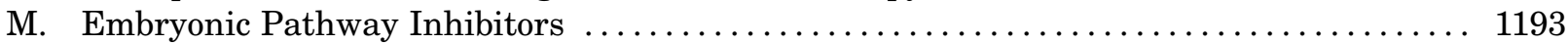

N. Histone Deacetylase Inhibitors: Reaching the Third Stage $\ldots \ldots \ldots \ldots \ldots \ldots \ldots \ldots \ldots \ldots$

IV. Target Exploration and the Drug-Target Network $\ldots \ldots \ldots \ldots \ldots \ldots \ldots \ldots \ldots \ldots \ldots \ldots \ldots \ldots \ldots \ldots$

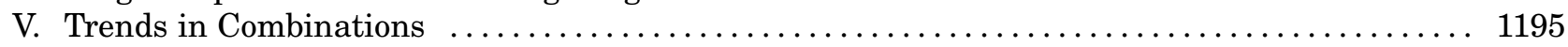

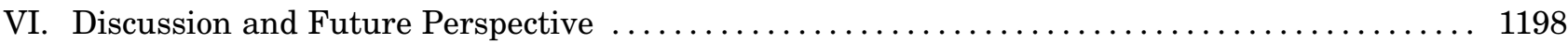

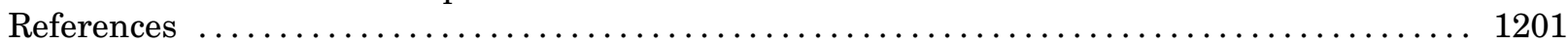

\begin{abstract}
Brain cancer is a formidable challenge for drug development, and drugs derived from many cutting-edge technologies are being tested in clinical trials. We manually characterized 981 clinical trials on brain tumors that were registered in ClinicalTrials.gov from 2010 to 2020 . We identified 582 unique therapeutic entities targeting 581 unique drug targets and 557 unique treatment combinations involving drugs. We performed the classification of both the drugs and drug targets based on
\end{abstract}

pharmacological and structural classifications. Our analysis demonstrates a large diversity of agents and targets. Currently, we identified 32 different pharmacological directions for therapies that are based on 42 structural classes of agents. Our analysis shows that kinase inhibitors, chemotherapeutic agents, and cancer vaccines are the three most common classes of agents identified in trials. Agents in clinical trials demonstrated uneven distribution in combination approaches; chemotherapy

Address correspondence to: Helgi B. Schiöth, Head, Unit of Functional Pharmacology, Department of Neuroscience, Uppsala University, Box 593, 75124 Uppsala, Sweden. E-mail: helgis@bmc.uu.se

This work has been supported by the Swedish Research Council, the Swedish Brain Foundation, the Swedish Cancer Foundation, and the Novo Nordisk Foundation.

The authors declare no conflicts of interest.

https://doi.org/10.1124/pharmrev.121.000317

[S This article has supplemental material available at pharmrev.aspetjournals.org. 
agents, proteasome inhibitors, and immune modulators frequently appeared in combinations, whereas kinase inhibitors, modified immune effector cells did not as was shown by combination networks and descriptive statistics. This analysis provides an extensive overview of the drug discovery field in brain cancer, shifts that have been happening in recent years, and challenges that are likely to come.

Significance Statement-This review provides comprehensive quantitative analysis and discussion of the brain cancer drug discovery field, including classification of drug, targets, and therapies.

\section{Introduction}

Brain cancer represents a significant challenge for current oncology and drug development. Despite being rare in the general population, malignant brain and CNS tumors account for up to $33 \%$ of cancerrelated deaths in children according to data from the United Kingdom from 2010 to 2012 (McNeill, 2016). The more recent worldwide statistics also suggest that brain and nervous system cancers are one of the most common cancer-related deaths in patients aged between 0-19 years and 20-29 years. (Fitzmaurice et al., 2018). The most common subtype of malignant tumors is malignant glioma, whereas the most aggressive subtype is represented by glioblastoma (McNeill, 2016). Brain tumors vary in terms of malignancy, and this results in significant differences in patient survival in all age groups. For example, the 5year survival rate of patients with nonmalignant brain tumor in all age groups is $91 \%$. However, tumor malignancy reduces this number to about $32 \%$ overall, and in case of glioblastoma, this number is much lower and is about 5\% (Barnholtz-Sloan et al., 2018).

The primary approach to treat cancer has been chemotherapy, but the treatment paradigm is increasingly shifting toward targeted therapeutic approaches. This development was manifested with the FDA approval of trastuzumab, an antibody targeting ERBB2 for the treatment of breast cancer, in 1998 and imatinib, an inhibitor of BCR-ABL kinase, for $\mathrm{Ph}+$ chronic myeloid leukemia in 2001 (Rask-Andersen et al., 2014) with many more kinase inhibitors being approved thereafter. Currently, targeted therapies are enriched by personalized and genome-driven methods (Hyman et al., 2017; Gambardella et al., 2020) guided by an active search for biomarkers (Sun et al., 2020). The last 10 years are also characterized by successful clinical validation of advanced immune and gene therapies, which is likely to prompt a broader application of new therapies. Importantly, neuro-oncology has become a testing field for cutting-edge therapies, which have emerged either specifically for brain tumors or to treat many other cancers. For instance, EPH receptor (EPH)ephrin signaling has been extensively studied over the years, which has led to the exploration of EPH inhibitors and EPH-targeting immune therapies in various conditions, including malignant glioma (Boyd et al., 2014). Another interesting example is oncolytic viral therapy, which is based on viral killing of tumor cells that can potentially be used either as monotherapy or in combinational approaches with immune therapy to enhance clinical performance in different malignancies (Twumasi-Boateng et al., 2018).

Several previous publications have provided the benchmark for analysis of drug discovery trends among different therapeutic areas, investigating overall pharmaceutical innovation; exploration of novel targets, for example, among kinases and G protein-coupled receptors (GPCRs); specifics of orphan drug development and approval; and pivotal directions of targeting within the human genome as well as approval trends (Munos, 2009; Rask-Andersen et al., 2011; DiMasi et al., 2013; Hauser et al., 2017; Santos et al., 2017; Attwood et al., 2018; Tambuyzer et al., 2020). Several detailed reviews exist tailored to a specific drug class or type of brain cancer [e.g., discussing metabolic targeting of the tumor metabolism with indolamine 2,3-dioxygenase (IDO) inhibition (Platten et al., 2019) and advances in brain tumor immune therapy (Sampson et al., 2020) as well as using targeted approaches in malignant glioma and medulloblastoma (Huse and Holland, 2010; Kumar et al., 2017)]. One quantitative analysis demonstrated that the adoption of temozolomide with radiation therapy as a standard-of-care regimen has led to a long-term increase in the overall survival of patients with glioblastoma multiforme. This publication also reported the highest survival rates in trials with bevacizumab, tumor treating fields

ABBREVIATIONS: AKT, protein kinase Akt; BBB, blood-brain barrier; BET, bromodomain and extraterminal motif; CAR, chimeric antigen receptor; $\mathrm{CB}$, checkpoint blockade; CDK, cyclin-dependent kinase; CIIM, checkpoint inhibitor and immune modulator; CNS, central nervous system; CTLA-4, cytotoxic T-lymphocyte-associated protein 4; DC, dendritic cell; EGFR, epidermal growth factor receptor; EPH, EPH receptor; FDA, US Food and Drug Administration; FGFR, fibroblast growth factor receptor; FLT, FMS-like tyrosine kinase; GM-CSF, granulocyte macrophage colony-stimulating factor; GPCR, G protein-coupled receptor; HDAC, histone deacetylase; HER2, human epidermal growth factor receptor 2; HIF2A, hypoxia-inducible factor $2 \alpha$;SV, herpes simplex virus; hTERT, human telomerase reverse transcriptase; IDH, isocitrate dehydrogenase; IDO, indolamine 2,3-dioxygenase; IL, interleukin; IL13RA2, interleukin-13 receptor $\alpha$ subunit; JAK, Janus kinase; MDM, E3 ubiquitin-protein ligase Mdm; MHC, major histocompatibility complex; MTOR, mechanistic target of rapamycin; NK, natural killer; PARP, poly(ADP-ribose) polymerase; PD1, programmed cell death 1 (or PDCD1); PCV, procarbazine, lomustine (CCNU), and vincristine; PD-L1, programmed death-ligand 1; PI3K, phosphoinositide 3-kinase; RTK, receptor tyrosine kinase; ScFv, single-chain variable fragment; STAT, signal transducer and activator of transcription; TCR, T-cell receptor; TTF, tumor treating field; VEGFA, vascular endothelial growth factor A; WT1, Wilms tumor gene-1. 
(TTFs), and vaccines (Marenco-Hillembrand et al., 2020). Today many new therapies are being tested in the clinic, and it is a major challenge to have an overview of the entire field. To our best knowledge, a comprehensive quantitative assessment that systematically covers clinical drug development in brain cancer trials, drugs, and drug targets has not yet been published. Here, we present an analysis of drug interventions to treat brain malignancies that have been in clinical trials during the last 10 years. We provide a detailed structural and pharmacological classification of these interventions as well as the characteristics of their targets. Furthermore, we analyze the combination therapies-drug target networks and discuss the trends in monotherapies and combinations to treat brain cancers.

\section{Data Collection and Analysis}

The clinical trial studies were initially downloaded from the ClinicalTrials.gov database. Search results were obtained using the following search terms: "Brain cancer," "Brain tumor," "Glioma," "Glioblastoma," "Astrocytoma," "Medulloblastoma," "Oligodendroglioma," "Pituitary tumor," "Ependymoma," "Meningioma," "Gliosarcoma," "CNS Atypical Teratoid Rhabdoid Tumor," "Gliomatosis Cerebri," "Pineal Region Tumor," "CNS Primitive Neuro-Ectodermal Tumors." These terms were applied to encompass all interventional trials with a drug or preparative therapy for the primary purpose of treatment with a study first posted date of January 1, 2010 or later. Other study designs might use the actual study start date instead of the date the trial appeared on ClinicalTrials.gov. We included clinical trials on all age groups in the analysis to capture more agents/therapies. The initial $\sim 5600$ studies (1930 without overlaps) were manually assessed, and studies on nonbrain tumors, such as schwannomas, were removed as well as brain metastases studies since these tumors possess a distinct molecular profile different from that of primary brain tumors. Furthermore, other studies were removed if they investigated an agent against tumor comorbidities (like edema) or if they did not include treatment arm descriptions or if the purpose of the study was imaging, for example, 5-aminolevulinic acid. Studies on low-grade primary brain tumors were left in the data since some of these tumors (such as low-grade gliomas) may transform into malignant forms (Claus et al., 2015). After manual curation, the total count of clinical trials was 981 unique studies.

To obtain data on monotherapies and combination therapies, we manually analyzed trial descriptions, interventions, and arm descriptions. It is important to make a distinction between the investigative arm and the controls, and here the control arms were not considered for this quantitative analysis. It should be noted that we did not consider different dosages, routes of administration of drugs, and treatment cycles as well as surgery and different types/amount of radiation therapy. Radiation therapy and tumor treating fields were, however, included in combinations but omitted if monotherapy.

We classified several biologicals within the same group [chimeric antigen receptor (CAR) T cells, CAR natural killer (NK) cells, dendritic cells, tumor lysate vaccine, stem cells, cytotoxic $\mathrm{T}$ cells] as different drug entities since there are many factors involved in their production. For example, anti-epidermal growth factor receptor (EGFR)-CAR T cells could be generated employing different initial $\mathrm{T}$ cells, transduction systems, receptor structure, culture medium, etc. We named biologicals with a single identical name only if they were manufactured in the same laboratory, the trial had the same principal investigator, and medication had a trade name (or code name) allowing it distinction from other biologicals with close structure. Some clinical trials had ambiguous phase descriptions, requiring correction for quantitative analysis. The following logic was used: Phase "NA" was treated as phase one, and trials with both phase one and phase two status were treated as trials of phase one, whereas trials with both phase two and phase three status were treated as trials of phase two. We made such substitutions because of uncertainty if the trial successfully transitioned to the next phase.

We manually classified drugs, using physical (structural) and pharmacological classifications. The priority was to make the classifications were mostly relevant to the oncology field. We did not use the chemical structure of small molecules as the basis for our classification since it is beyond the scope of this publication. Genetic modification of immune cells was considered as a gene therapy according to the FDA website (https://www.fda.gov/vac cines-blood-biologics/cellular-gene-therapy-products/whatgene-therapy). To obtain drug targets, we used the DrugBank database, updated data from our previous publication (Rask-Andersen et al., 2011), PubMed searches, manufacturer's websites, and public sources. We manually classified targets using the UniProt database and PubMed searches. The enzymes were classified in accordance with the biologic function of a particular enzyme. Kinases were specified as a separate class because of their crucial role in cancer biology. The Transporter Classification Database was used to classify transporters (Saier et al., 2014). To analyze the dataset, we used the $\mathrm{R}$ programming language with the following packages: ggplot2, dplyr, xlsx, igraph, stringr, networkD3, and visNetwork. These packages are a part of the Comprehensive $R$ Archive Network and can be downloaded from its repository. For supplementary network images we used the Gephi software.

To analyze trends in drug classes, we used the metric "presence" instead of the number of clinical trials. The presence was calculated using the combined 
number of all drug entities of a particular class per year. If a particular drug appeared in a clinical trial, it was counted only once, no matter the number of arms with this agent in a trial. However, if there are two or more agents of the same class in the same trial, this trial is counted twice or as many times as the number of agents of the class in the trial. One clinical trial can investigate two innovative agents of the same class at once. For instance, one trial can analyze two kinase inhibitors simultaneously either in different arms or in combination. The metric "number of trials" gives the result of 1 , whereas the "presence" gives the result of 2 . Using presence instead of a number of the clinical trials allows detecting trends more accurately.

\section{Overall Trends in Drugs, Drug Targets, and Biologic Therapies}

Overall, 582 therapeutic modalities have been investigated from 2010 till the beginning of 2020 . These entities have been appearing fairly uniformly throughout the years (Fig. 1A), and the proportion of the different clinical trial phases remained similar each year during our analysis (Fig. 1B). The peak of 2010 could be explained by the fact that some of the agents had already entered trials before and were included in 2010 since our timeframe starts from this year.

In our analysis, we used structural and pharmacological classifications to describe therapeutic entities (Fig. 2). The structural classification relies on a physical structure of a particular molecule, complex, cell, or virus. Identifying the structural classification is important because of its tight nexus with manufacturing technologies of drugs and their pharmacokinetics. The key features and structures of core structural classes are outlined in Table 1 and Fig. 3 (extended version is in Supplemental Table 1). Pharmacokinetics is a major concern in brain cancer therapy since the blood-brain barrier (BBB) prevents polar, lipid-insoluble, or large molecules ( $>600 \mathrm{Da}$ ) from entering a brain (Scherrmann, 2002). From a structural perspective, most of the interventions are small molecules (309 agents, $\sim 53 \%$ ), which could be related to well established pipelines to manufacturing and favorable absorption, distribution, metabolism, and excretion properties of small molecules. However, often even small molecules do not demonstrate a desired distribution within an organism, thus requiring the use of delivery systems, which often carry chemotherapy agents. Overall, there are 15 various small molecules employing delivery systems. These systems include metal nanoparticles, liposomes, polymer nanoparticles, protein (albumin) nanoparticles, and a bacterial-derived carrier.

Monoclonal antibodies are another abundant drug structure in clinical trials (42 agents; 7\%). This class of molecules has pharmacokinetic issues, in particular, penetration into the tumor tissue (Thurber et al., 2008). Several modified versions of monoclonal antibodies, like antibody-drug conjugates, chimeric proteins, bispecific antibodies, and peptibodies were identified in trials, although these modifications are relatively rare compared with regular antibody products. Various cell products, including CAR T cells (34 entities; 5.8\%) and dendritic cells (35 entities; 5.9\%), were also often examined in the clinic especially in last years, thus showing a rising interest in such kinds of therapies. Additionally, many protein and peptide formulations that are primarily used as vaccines are found in trials. Thirty-six clinical trials have been analyzing the use of viruses both for gene therapy and for tumor lysis. In a gene therapy, these viruses frequently contain some introduced gene, such as encoding cytokines like interleukin 12 or ligands that can facilitate an antitumor immune response or induce apoptosis in tumor cells.

In addition to the structural classification that enables identifying trends in technological platforms, we used the pharmacological classification that is related
A

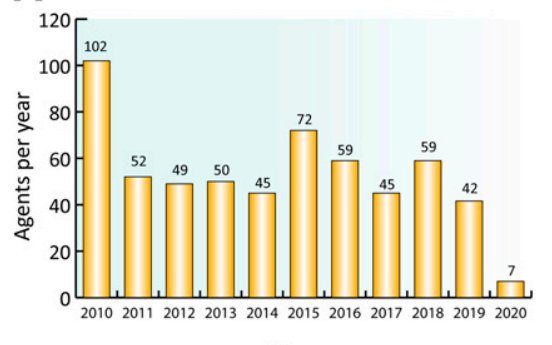

Year
B

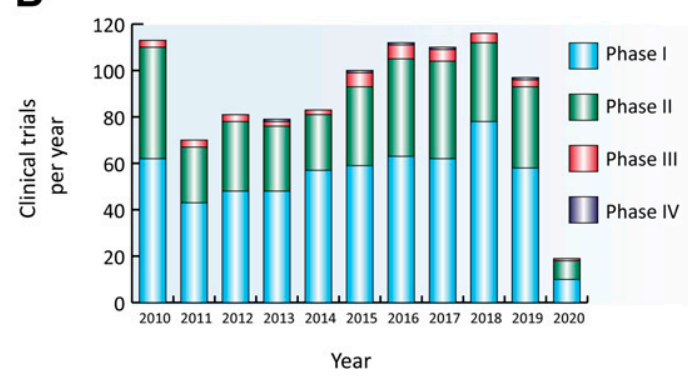

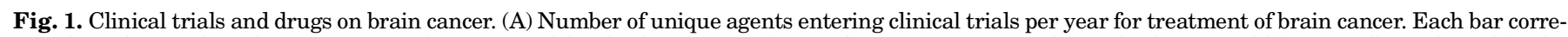

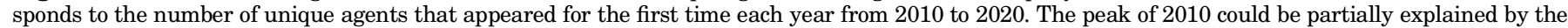

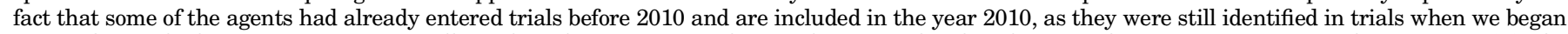

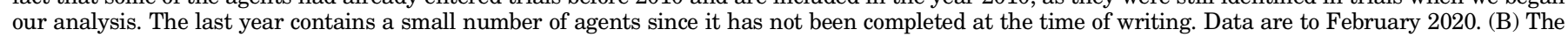

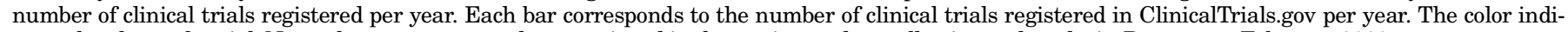
cates the phase of a trial. Note, phases were treated as mentioned in the section on data collection and analysis. Data are to February 2020. 


\section{Unique therapeutic entities}

Structural classification

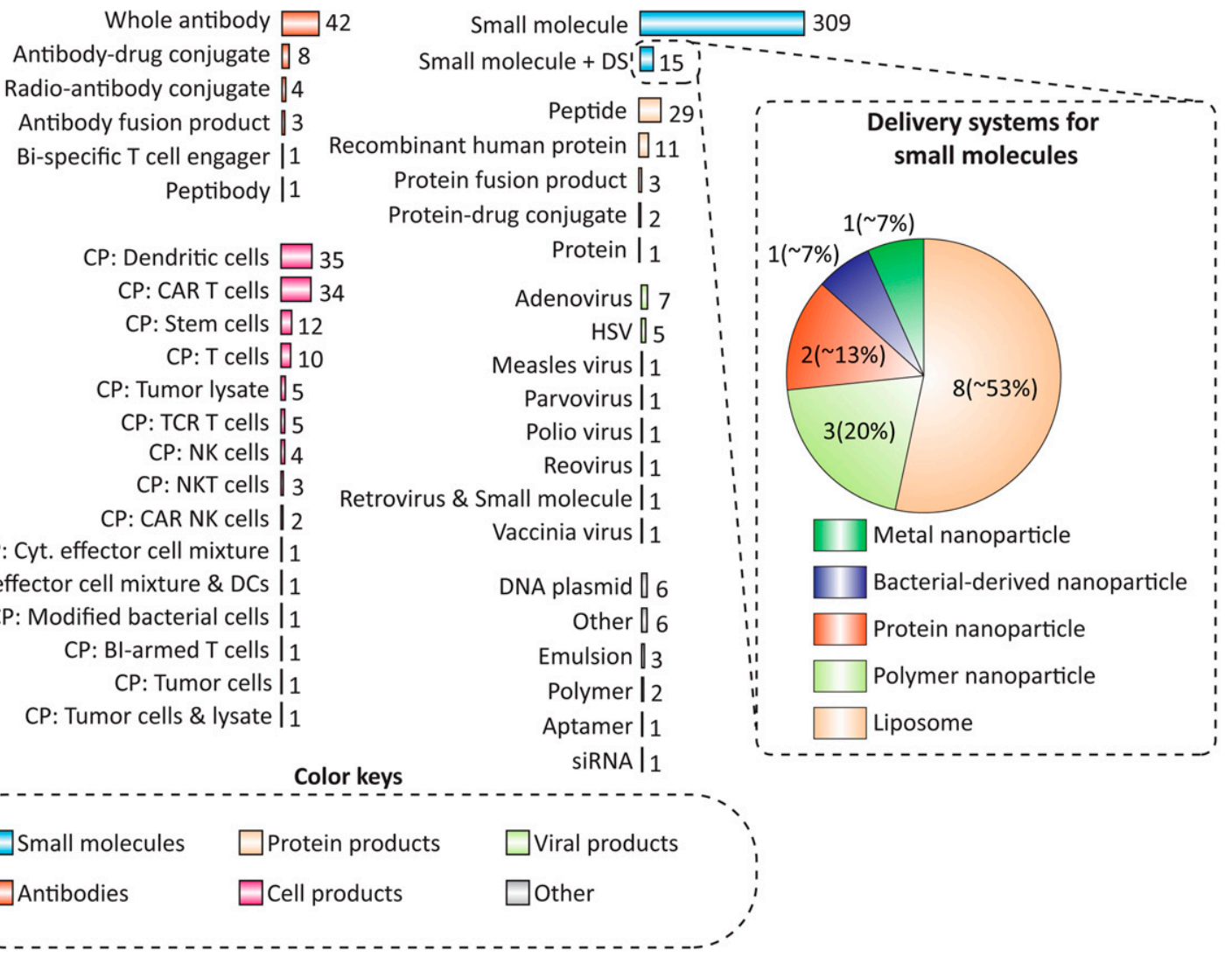

Fig. 2. Structural platforms in brain cancer trials. The structural classification of the drug agents. The structural classification of the 568 drug entities identified in the analysis are separated into six superclasses. Some of the entities represent named combinations, thus their structure is shown using "\&." The size of a bar corresponds to the number of agents in the class. Each classification has color codes to illustrate the superclasses of entities. The right side of the figure details the diversity of delivery systems for small molecules in the data. Data are to February 2020. BITE, bispecific T-cell engager; CP, cell product; Cyt., cytotoxic; DS, delivery system; NKT cell, natural killer T cell; siRNA, small interfering RNA.

to treatment strategies to identify clinical trends (Fig. 4). Kinase inhibitors account for $15.6 \%$ of unique drugs investigated in clinical trials on brain cancer since 2010. These medications have been successfully used for other cancers, and it is not surprising that they are the most investigated therapeutic agents. The second major class is represented by cancer vaccines $(\sim 11.5 \%)$. Other significant directions include different kinds of chemotherapy, immune therapy, gene therapy, and targeted therapies. Generally, the pharmacological classes show much more even distribution when compared with the classification of the data based on drug structures, in which one class (small molecules) significantly outnumbers the other classes. Notably, both drug structures and pharmacological classes did not appear uniformly throughout the years, and this is further detailed below.

\section{A. Trends in Drug Targets}

Many drugs have a defined set of drugged proteins that are important during a broad overview of the drug discovery process. During our analysis, we identified 581 unique drug targets, including protein isoforms. The term target cannot be applied to all entities in our database. For example, the term "target" seems to be inappropriate for oncolytic viruses or hematopoietic stem cells. Thus, the number of agents used to gather targets was smaller than those of the entire dataset and equals 518 drug entities. In our analysis, we did not include drug targets whose pharmacological action designation was "NO" according to the drug bank since there is a high confidence that such a target is not a basis for pharmacological action. However, it must be mentioned that anticancer agents may exhibit their activity not through an initially proposed target (Lin et al., 2019). Thus, we decided not to remove other targets.

The locations of most targets $(40.4 \%)$ correspond to cellular membrane, which is followed by cytoplasm (26.9\%) and nucleus/nuclear envelope (15.7\%) (Fig. $5 \mathrm{~A})$. The percentage of membrane-localized targets 


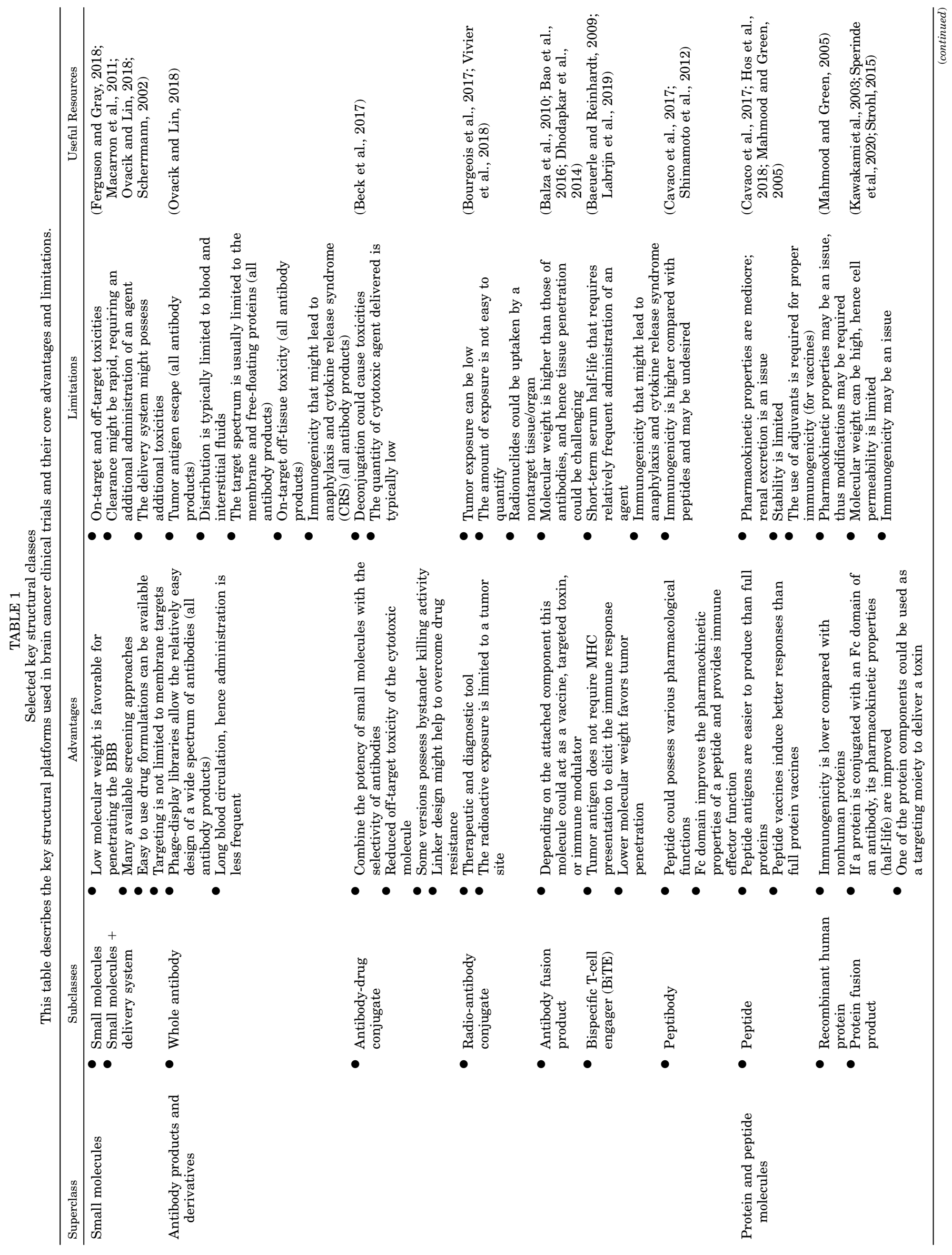

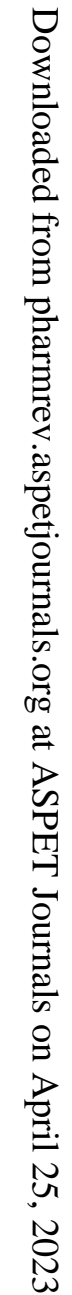



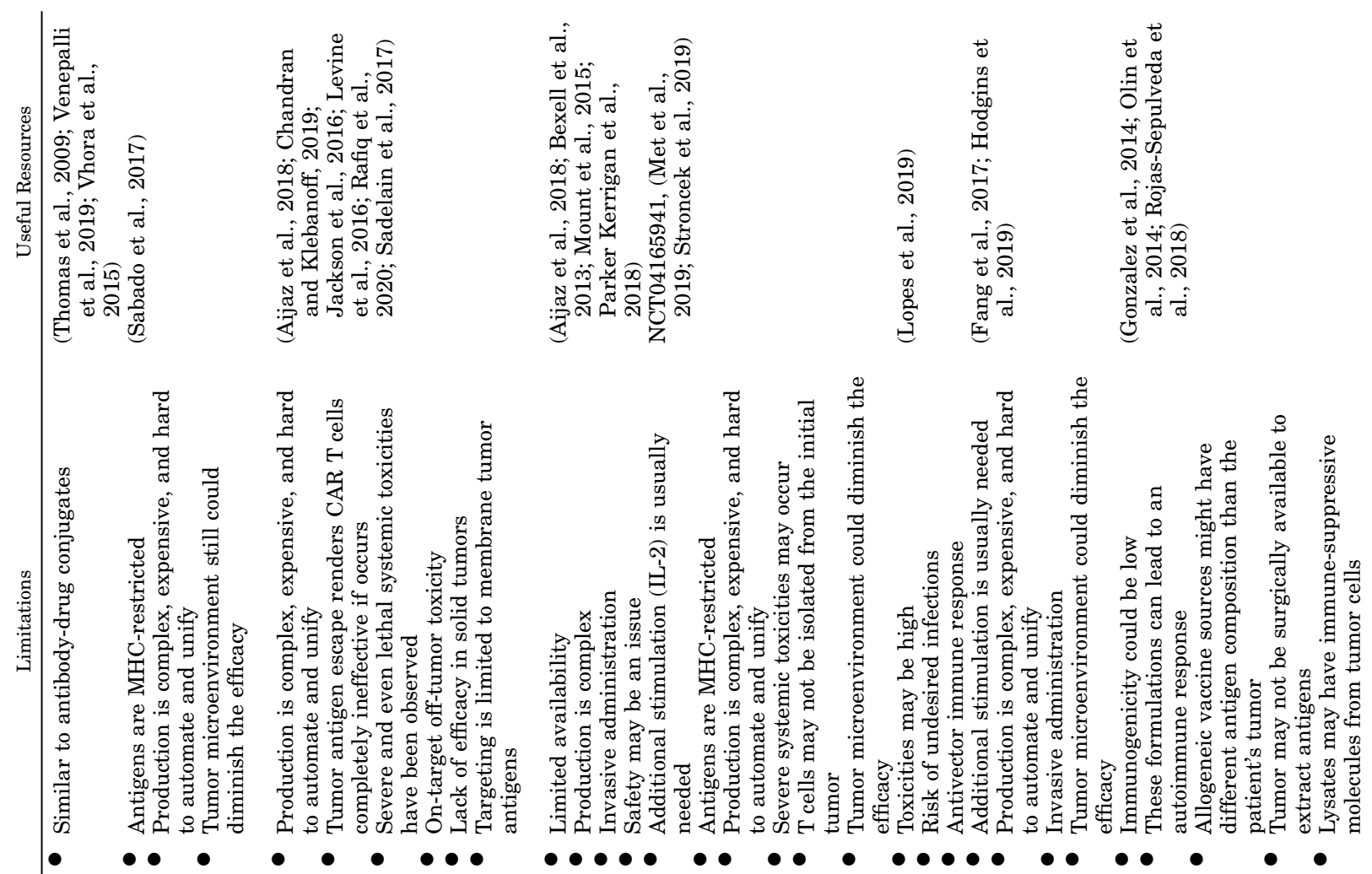

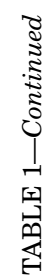<smiles>CCCCCCCC</smiles>

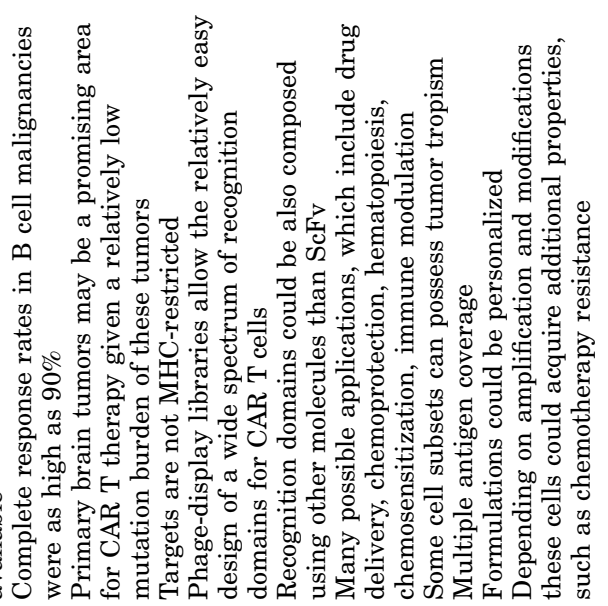
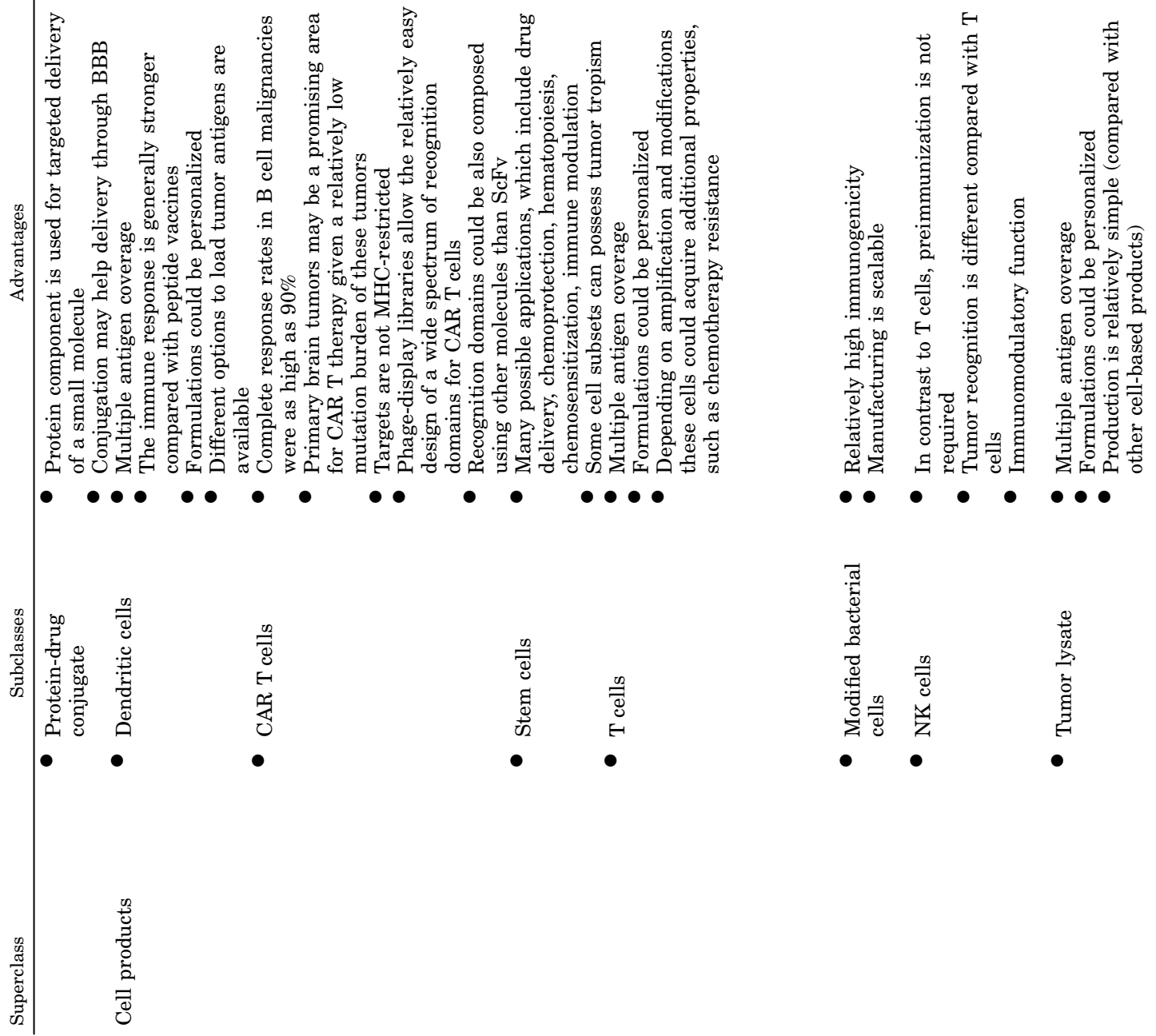


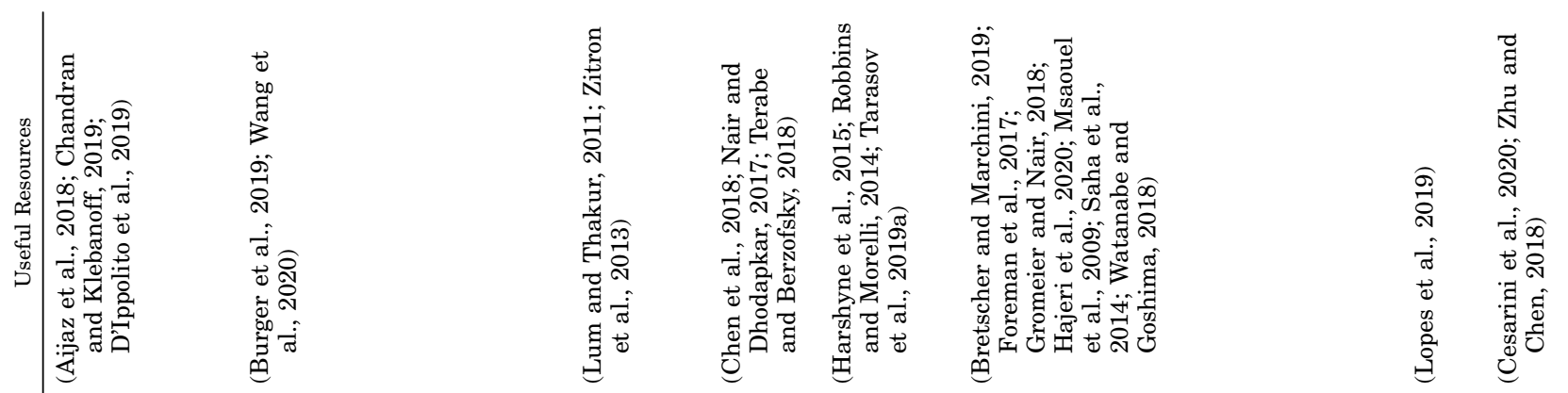
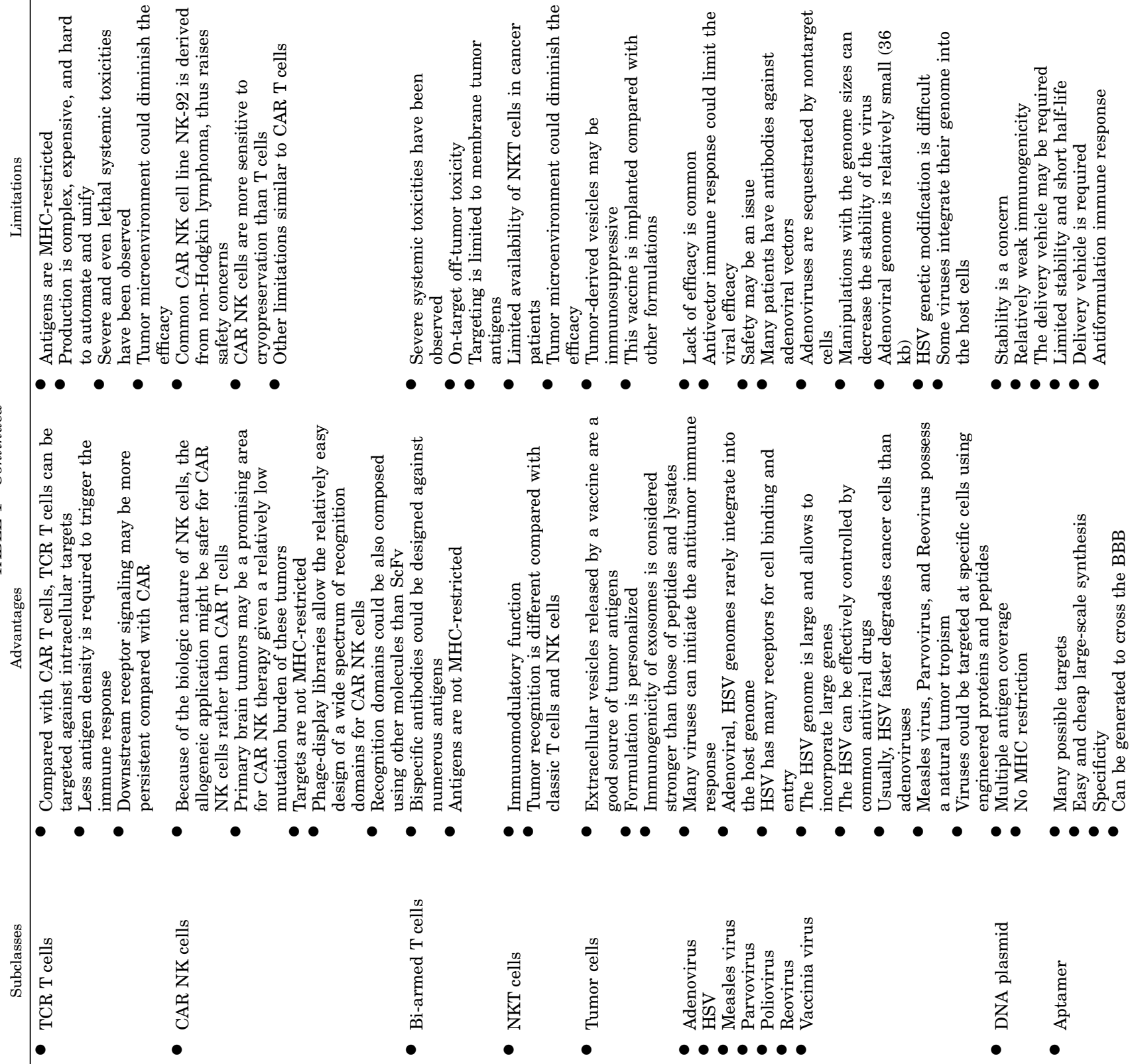


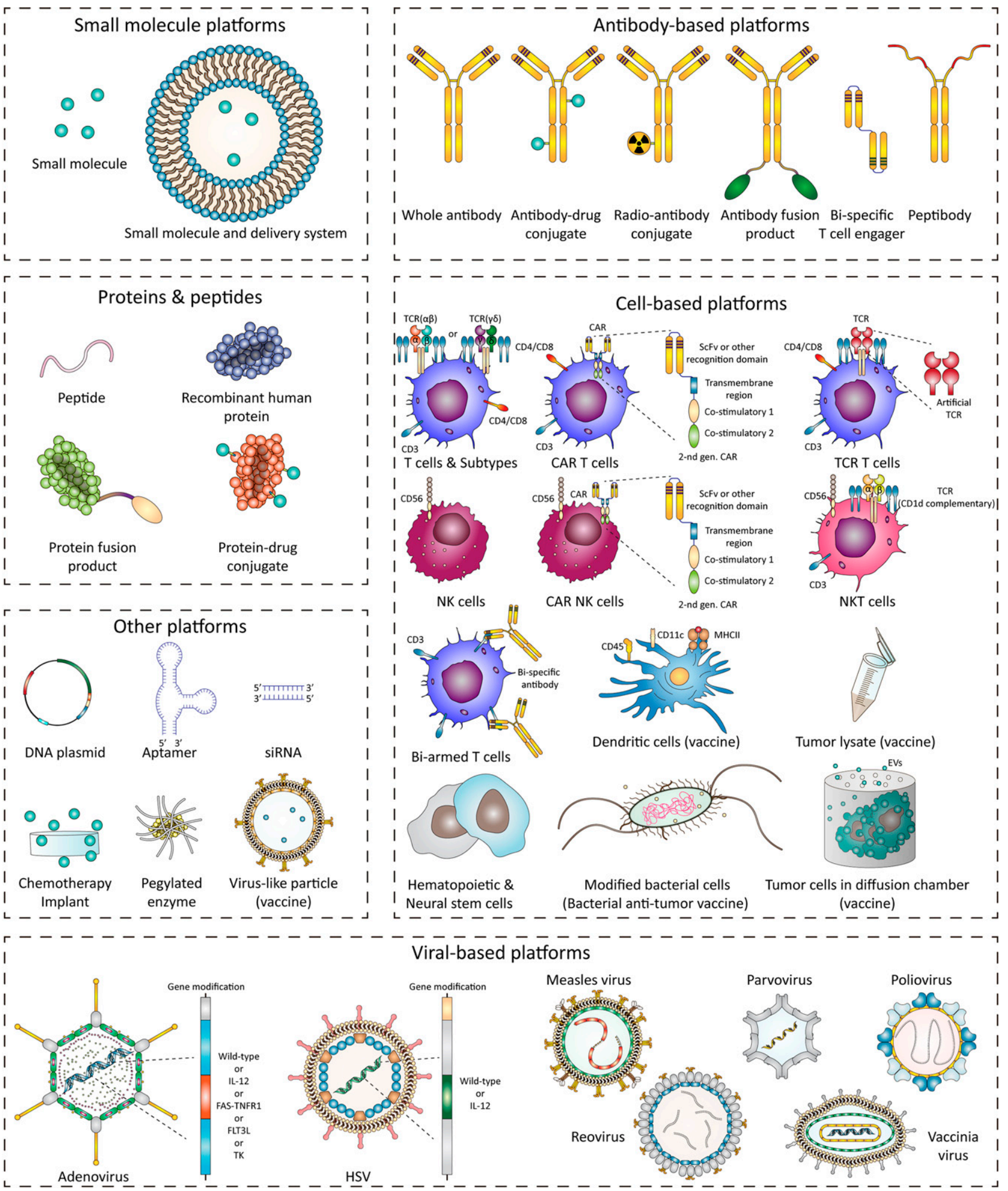

Fig. 3. Key structural platforms. Key structural platforms and classes of agents. This figure provides a graphical representation of the key structural classes identified in clinical trials on primary brain malignancies. Small-molecule platforms may contain delivery systems that could be of different origins. Antibody-based platforms contain bispecific T-cell engager therapies wherein the domains bind with different antigens. In several cell-based platforms, the key cell-surface markers that are used to sort these cell types were illustrated. T cells could possess different versions of a T-cell receptor$\alpha \beta$ or $\gamma \delta$, depending on a subtype. TCR T cells use an artificial T-cell receptor that is typically composed of a single protein chain. The TCR of the NKT cells is considered to be CD1d complementary and response to lipid antigens. The 2nd generation CAR structure for CAR NK and CAR T cells was used since it is the most common form of this construct. Dendritic cells are used as a vaccine after pulsed with tumor antigens. Bacterial cells are used to express tumor peptides and deliver them to immune cells to trigger the antitumor immune response. Viral-based platforms include different types of viruses that can be used in different strategies, such as adenovirus and HSV that are both used as oncolytic viruses or as a vector for gene therapy. Their genomes could be genetically edited to insert a gene of interest that could activate the immune response, trigger the apoptosis of tumor cells, or confer sensitivity to chemotherapy. Viruses are illustrated schematically and may not represent the actual structure in detail. FAS-TNFR1, chimeric FAS and tumor necrosis factor receptor 1; NKT cell, natural killer T cell; siRNA, small interfering RNA; TK, thymidine kinase. 


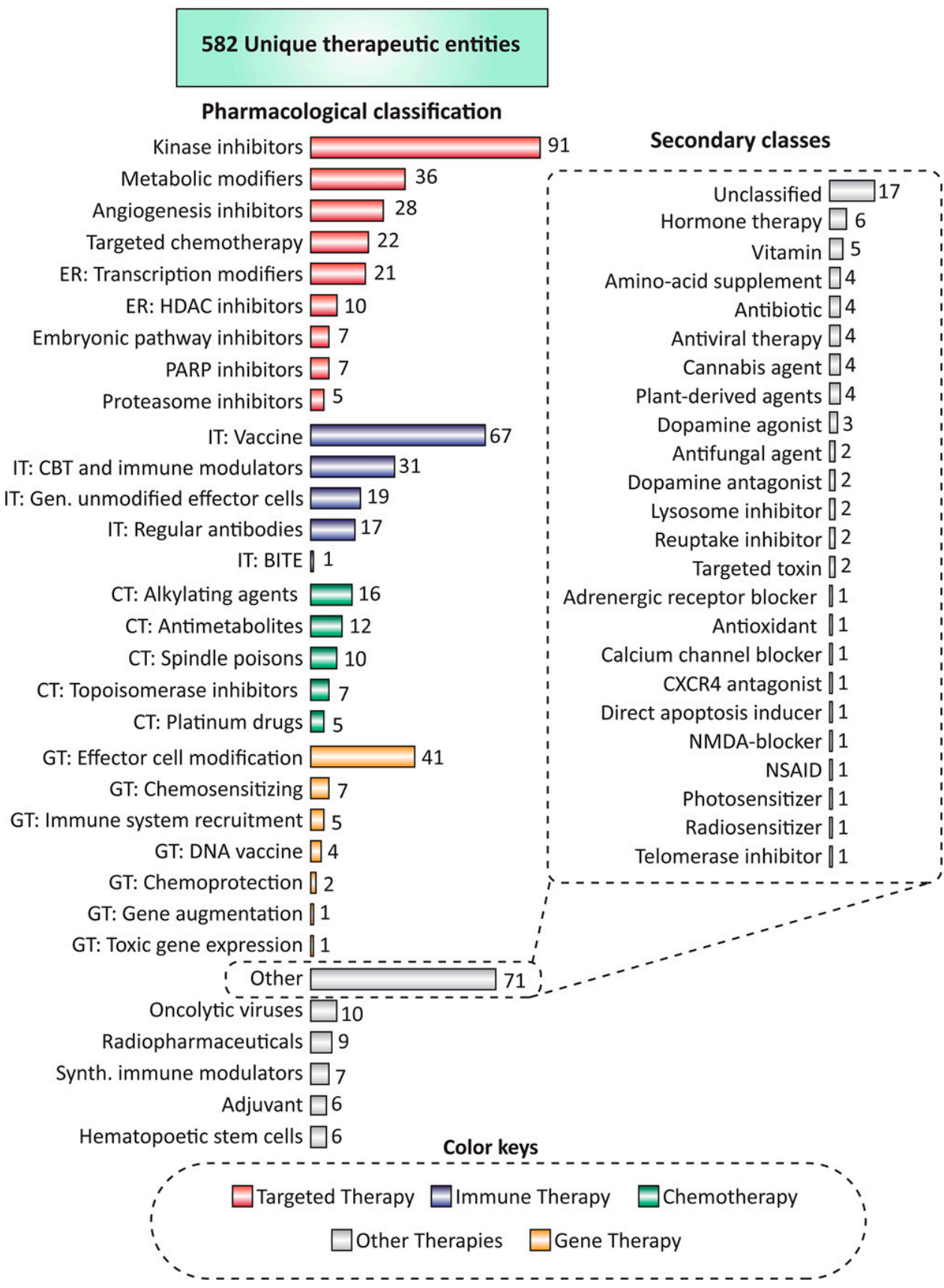

Fig. 4. Pharmacological directions of therapies. The pharmacological classification of the drug agents. The 568 drug entities identified in the analysis are classified by pharmacological classes into five superclasses, which are colorcoded. Information was obtained using cancer.gov, fda.gov, and PubMed search. Note that if a kinase inhibitor exhibits very clear antiangiogenic activity, it is classified as angiogenesis inhibitors. The right side of the figure provides secondary classification for other therapies (that has not been used for trend analysis). Data are to February 2020. By the term "genetically unmodified effector cells" we mean cells with unmodified immune effector function. BITE, bispecific T-cell engager; CBT, checkpoint blockade therapy; CT, chemotherapy; CXCR4, C-X-C chemokine receptor type 4 ; ER, expression regulator; Gen, genetically; GT, gene therapy; IT, immune therapy; NMDA, $\mathrm{N}$-methyl-D-aspartate receptor; NSAID, nonsteroidal antiinflammatory drug; Synth., synthetic. aligns well to the previous analysis undertaken by Yildirim et al. (2007). Other locations are less frequent and account for $17 \%$ of the drug-target spectrum combined. Such results are not surprising, considering that many targeted therapies for cancer act on tyrosine kinases that either are receptors and are located on the cellular membrane or represent a cytosolic protein that could be found both in the cytoplasm or nucleus, depending on a particular enzyme. Many other proteins, including enzymes, such as histone deacetylases (HDACs) or poly (ADPribose) polymerase (PARP), also demonstrate similar localization and could be found either in the cytoplasm or nucleus.
Using structural and functional properties to classify the targets shows that enzymes are the most abundant target class with almost $41.8 \%$ of all targets, and receptors are the second most common class and account for $\sim 31.5 \%$ (Fig. $5 \mathrm{~B}$ ). More specific characterization of the drug targets shows that kinases are the most targeted direction among enzymes as well as all targets (Fig. 5C). The second most commonly targeted enzyme class is represented by metabolic enzymes. Receptors are the third most investigated targets that include receptor tyrosine kinases (RTKs), GPCRs, and immune-related receptors. Interestingly, in comparison with enzymes and receptors, the pools of 


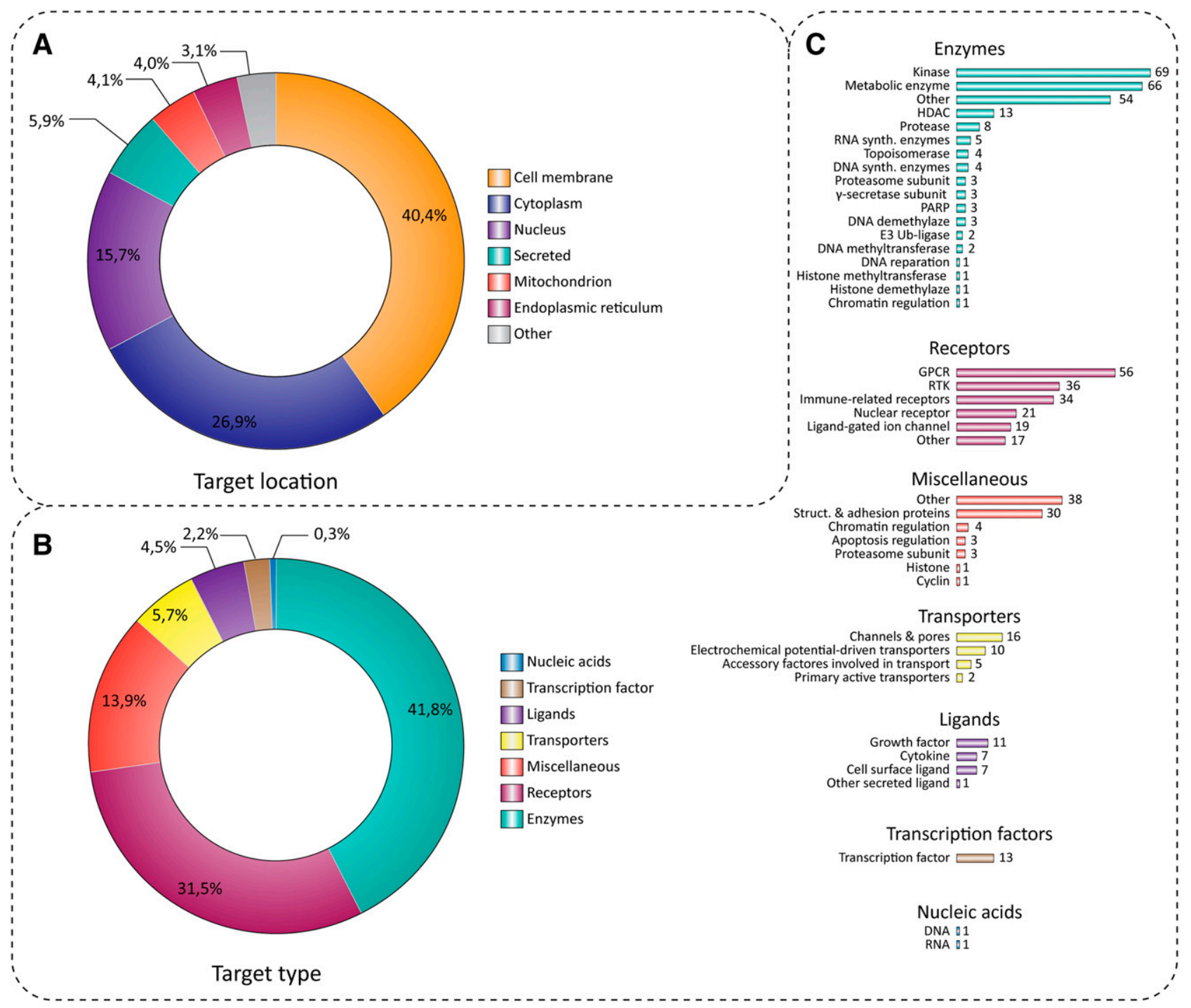

Fig. 5. Targets and their distribution. (A) The distribution of target locations. Each target location was manually obtained from the UniProt database and PubMed search. If a target had multiple cellular localizations, we included the most relevant for its functional activity. If the exact function is unknown or hard to determine, we used the location with the largest number of references. Color corresponds to a target location. (B) The general functional activities of the drug targets. Each drug target was manually classified using the UniProt database and PubMed searches. Targets are colorcoded corresponding to seven major functional classes. (C) Specific descriptions of each class of drug target presented in part B. Transporters were classified using the Transporter Classification Database. The term "kinase" refers to all kinases, excluding RTK and nontyrosine receptor kinases. Struct., structural; Synth., synthesis.

ligands, transporters, and transcription factors are relatively small.

It should be specified that the abovementioned figures represent a qualitative perspective of the target spectrum in our analysis. We visualized trends for each target class (see additional supplementary materials). Targets demonstrated trends similar to their corresponding drug classes. Interestingly, isoforms of HDACs and vascular endothelial growth factor A (VEGFA) demonstrated steady downward trends, whereas programmed cell death 1 (PD1 or PDCD1) and cytotoxic T-lymphocyte-associated protein 4 (CTLA-4) that are the targets for checkpoint inhibitors showed very noticeable up-trending behavior. Several kinases, including deoxycytidine kinase, BRAF, and Lim kinase also showed increasing presence during the years. Many targets showed no trends and appeared in a very limited number of trials.

\section{B. The Basics, Chemotherapy}

Chemotherapy is a staple of cancer treatment, and its primary action is based on cytotoxic effects resulting in cell death. Antimetabolites are the chemotherapeutic agents wherein the primary action hinges on substituting the cellular substrates with the drug, thus resulting in the inhibition of the cellular growth and cell cycle arrest. To date, no antimetabolites have been approved for brain cancer by the FDA. Since 2010, the presence of antimetabolites applied alone or in combination has increased 
in clinical trials, peaking in 2016 and 2018 with eight trials in each year, respectively. The most common drugs include azacitidine, fludarabine, gemcitabine, and methotrexate. Interestingly, azacitidine has been discovered to possess hypomethylating properties and has been investigated in brain tumors, including isocitrate dehydrogenase (IDH) 1/2 mutated specimens (Federici et al., 2020). The important feature of IDH1 is that it is a prognostic factor of patient survival in gliomas (Sanson et al., 2009), and two of seven clinical trials with azacitidine have the IDH status specified in the inclusion criteria (NCT03684811, NCT03666559). Decitabine is another drug that demonstrated a hypomethylating activity similar to azacitidine in hematologic tumors (Derissen et al., 2013). In brain cancer, this agent has been investigated twice - either in combination with dendritic cell (DC) vaccine (NCT02332889) or with cedazuridine (NCT03922555). The IDH status was noted in the inclusion criteria of the latter trial. The rationale of using decitabine with the DC vaccine is the property of this drug to enhance the expression of NY-ESO-1 antigen in gliomas (Konkankit et al., 2011). This trial, however, was discontinued due to enrolling only one participant (NCT02332889).

Another antimetabolite, fludarabine, was examined 15 times in 13 clinical trials on brain tumors since 2010 and always in combination therapies. It was used in combination with cellular therapies like CAR $\mathrm{T}$ cells and T-cell receptor (TCR)-modified T cells; it also appeared frequently with cyclophosphamide, an alkylating agent. A combination of fludarabine with cyclophosphamide represents a lymphodepletion regimen, and it is believed to favor the adoptive T-cell therapy (Salem and Cole, 2010). For instance, the addition of fludarabine to the lymphodepletion chemotherapy improved anti-CD-19-CAR T-cell persistence and survival of patients with blood cancer. Particularly, 16 of 17 patients who received cyclophosphamide with fludarabine and anti-CD-19-CAR T cells demonstrated a complete response (Turtle et al., 2016). Apparently, the high presence of fludarabine is explained by a growing interest in cellular therapies, in particular, CAR T cells and TCR T cells. Another agent, gemcitabine, has been analyzed in six different clinical trials as a part of combination therapies with kinase inhibitors, filgrastim, PARP inhibitors, or chemotherapy. Methotrexate is an antifolate drug that appeared in eight different clinical trials on brain tumors. Mostly, it has been combined with other chemotherapeutic medications.

Alkylating agents, another branch of chemotherapy, represent one of a few drug classes that embed the approved drug for brain cancer therapy. These drugs provide cellular cytotoxic effect via crosslinking DNA molecules, causing double-strand breaks, hampering uncoiling, and leading to the apoptosis of a cell. Sixteen drugs have entered clinical trials since 2010 as part of the alkylating agents class, and three of these medicines-temozolomide, cyclophosphamide, and lomustine-were the most frequently applied. In the United States, temozolomide was authorized for use in patients with brain cancer for the first time in 1999, and in 2005, a combination of temozolomide with radiation therapy was approved for newly diagnosed glioblastoma multiforme (https://www.access data.fda.gov/scripts/cder/daf/index.cfm?event=overview. process\&ApplNo=021029). According to our estimations, temozolomide is the most abundant drug in the whole field and has been tested in 275 different clinical trials in the last 10 years. This alkylating agent has been examined in 209 unique combinations even without taking into account various types of radiation therapy. In the last 10 years, the most common combinations of this drug were with radiation therapy and bevacizumab. Cyclophosphamide is the second most investigated alkylating agent that has been examined in 39 different clinical trials in 48 unique combinations. Before 2016, it was used as a part of combinational chemotherapy regimens to treat brain tumors. Then, with the expanding popularity of cellular therapies, it started to appear in combination with CAR T cells and TCR T cells as a primary component of the lymphodepletion regimen with or without fludarabine.

The third most common alkylating agent identified in our database is lomustine (or CCNU). It has been examined in 21 unique combinations in 24 trials since 2010. Compared with cyclophosphamide, the high presence of this drug is not influenced by the growing popularity of cellular products. In fact, we found no trials investigating the concomitant application of any kind of cell product with lomustine in patients with brain cancer. Initially, lomustine was approved by the FDA for brain tumors and Hodgkin disease in 1976 (https://www.accessdata.fda.gov/drugsatfda_docs/ label/2016/017588s043lbl.pdf). This agent is used as a part of a prominent combinational chemotherapy regimen, which is called procarbazine, lomustine (CCNU), and vincristine (PCV), with procarbazine (another alkylating agent) and vincristine. According to several clinical trials, lomustine demonstrates better results when it is a part of the PCV regimen, especially if used in patients with $1 \mathrm{p} / 19 \mathrm{q}$-codeleted oligodendroglioma (Weller and Le Rhun, 2020). In our data, however, we detected the PCV regimen only in three trials. A phase three POLCA trial started in 2015 is currently investigating $\mathrm{PCV}$ in anaplastic gliomas with $1 \mathrm{p} / 19 \mathrm{q}$ codeletion (NCT02444000). We also would like to note another alkylating agent, carmustine. Injectable carmustine was approved in the United States for brain tumors in 1977 (https://www.accessdata.fda.gov/drugs atfda_docs/label/2017/017422s055lbl.pdf). Interestingly, we did not identify studies with injectable form of the 
drug during the analyzed time span. The interesting feature of carmustine is that this drug has been examined as a carmustine sustained-release implant wafer (CASANT wafer) and as a GLIADEL wafer in the last 10 years in five different clinical trials. The purpose of these two implants is to provide prolonged release of carmustine directly into a patient's surgical cavity after tumor resection. FDA authorized GLIADEL for use in gliomas in adjunction to surgery or radiation therapy in 1996 (https://www.accessdata.fda.gov/drug satfda_docs/label/2018/020637s029lbl.pdf). Interestingly, we did not find studies with carmustine after 2014, and of five studies presented in the dataset, only one has been completed. In this study, the GLIADEL administration after 5-aminolevulinic acid-supported tumor resection resulted in 15 months of median overall survival of patients with glioblastoma, albeit not without relatively frequent (44\%) serious adverse effects (NCT01310868).

Another prominent class of chemotherapy agents is topoisomerase inhibitors. Topoisomerases are vital for enzymes in human cells that regulate DNA supercoiling, thus providing cellular homeostasis during transcription and DNA replication. Generally, there are two types of topoisomerases, type one and type two, which provide DNA uncoiling in a slightly different manner. Both of the two topoisomerase classes are targeted by topoisomerase inhibitors (Pommier, 2013). Seven drugs that could be classified as topoisomerase inhibitors were identified in brain cancer clinical trials since 2010. Irinotecan was the most investigated topoisomerase inhibitor in our database. This is a prodrug, the active metabolite of which, SN-38, acts via inhibiting type one topoisomerases. Irinotecan has been analyzed in 18 combinations in 19 different clinical trials, and the most common combinations of the compound were with temozolomide, kinase inhibitors, and bevacizumab. Additionally, we identified five clinical trials that investigated liposomal and nanoparticle formulations of irinotecan (NCT02022644, NCT02433392, NCT02481960, NCT03086616, NCT03119064). Etoposide is the second most investigated topoisomerase inhibitor that appeared in 26 unique combinations in 18 different clinical trials. This drug acts via inhibiting type two topoisomerases. Interestingly, in comparison with irinotecan, etoposide was combined mostly with chemotherapies (carboplatin, cisplatin, cyclophosphamide) and kinase inhibitors but only appeared once with bevacizumab. Topotecan is the last drug that was investigated relatively frequently in nine trials. Just like irinotecan, topotecan metabolizes into its active metabolite SN-38. Topotecan was applied either as monotherapy or in combination with pazopanib (angiogenesis inhibitor), alisertib (kinase inhibitor), and other agents.

\section{Gene Therapy Is Found in Six Classes}

Gene therapies are frequently investigated in patients with cancer. We identified six classes of gene therapy in brain tumors: modification of immune effector cells, chemosensitization of tumor cells, immune system recruitment to tumor cells, DNA vaccines, chemoprotection of normal cells, normal gene augmentation, and toxic gene expression in tumor cells. Two of these classes, effector cell modification and DNA vaccines, in turn demonstrated a growing interest from investigators in the last 10 years. Effector cell modification is an introduction of an artificial gene or gene editing of a natural gene in immune effector cells to facilitate the immune response against the tumor. This class includes modifications of immune effector cells, such as CAR T cells, TCR T cells, CAR NK cells, and others.

CAR T cells, along with checkpoint inhibitors, are probably the major breakthroughs in current cancer therapies. Currently, CD19 CAR therapy demonstrated outstanding clinical performance in patients with acute lymphoid leukemia (Sadelain et al., 2017). In one study, the complete response rate in 29 participants was very high at $93 \%$ (Turtle et al., 2016). Such impressive results led to the initiation of similar clinical trials in other cancers. Only a single phase one trial investigated CAR $\mathrm{T}$ cells in patients with brain cancer in 2010. In contrast, seven clinical trials on CAR T cells have been initiated in 2019. The main idea behind CAR T-cell therapy is an introduction of CAR into a patient's T cells, propagation of cells in vitro, and subsequent infusion of these cells into a patient to eradicate the tumor. CAR structure includes a binding domain [usually single-chain variable fragment $(\mathrm{ScFv})]$ to recognize a tumor antigen, hinge and transmembrane regions, and signaling domains. CAR structure could vary in terms of signaling domains, and hence there are three CAR generations. Basically, CAR T-cell design, manufacturing, and application are complex and involve rigorous considerations of the binding domain, target selection, signaling domain selection, transmembrane region optimization, gene delivery vehicle, population of $\mathrm{T}$ cells, cultivation, and safety. Discussing these factors is beyond the scope of this publication; to see more detailed information on CAR T cells see these comprehensive reviews (Jackson et al., 2016; Sadelain et al., 2017; Rafiq et al., 2020). In brain tumors, we identified several targets for CAR T-cell therapies: mutant EGFRvIII, Cluster of Differentiation 276 (B7-H3), prominin-1 (CD133), EPHA2, ganglioside G2 (GD2), human epidermal growth factor receptor 2 (HER2), mucin 1, and interleukin-13 receptor subunit $\alpha-2$ (IL13RA2). We also identified single trials investigating CAR T cells against basigin (CD147), EGFR (wildtype), EGFR806, and PD1. In two trials, CARs were 
targeted using either the chlorotoxin peptide (NCT04214392) or a fragment of the NKG2D receptor from NK cells (NCT04270461). An interesting CAR design that entered clinical trials involved using PD1 as a binding domain to switch the immune inhibitory signal from programmed death-ligand 1 (PD-L1) into activating signaling (NCT02937844). All of the aforementioned antigens have demonstrated their role in brain cancer development and progression. For instance, EGFRvIII, a mutated version of the epidermal growth factor receptor, is the most common oncogenic mutation found in glioblastoma multiform (An et al., 2018). The important and not yet resolved issue is whether the targets listed are suitable for CAR T cells in the clinical settings. In general, we identified 34 different clinical trials investigating CAR cells, and all of them were phase one trials. CAR cells were used 12 times as combinations and 28 times as monotherapies. Most of the trials with CARs did not have a lymphodepletion regimen or did not specify it in the trial description. Several CAR cell designs were derived from memoryenriched T-cell populations since this approach is believed to provide better antitumor responses (Sadelain et al., 2017).

TCR T cells represent an alternative to CAR cells. This approach relies on transferring the specific T-cell receptor gene into recipient $\mathrm{T}$ cells to provide an immune response against a desirable antigen. This method requires smaller amounts of antigen on tumor cells for T-cell activation; however, antigens for this therapeutic class are MHC-restricted and not always amenable for targeting. But this is contrasted by the fact that TCR engineering allows targeting intracellular proteins, of which fragments can be presented on MHC molecules (Chandran and Klebanoff, 2019). Unfortunately, TCR $\mathrm{T}$ cells failed to demonstrate a more favorable safety profile, as one might expect, over CAR $\mathrm{T}$ cells in solid tumors, given that severe toxicities were reported from several clinical trials on different tumor types (D'Ippolito et al., 2019). Currently, only six clinical trials are investigating TCR T cells in brain tumors, with three of these being phase two. In contrast to CAR T cells, TCR $\mathrm{T}$ cells were found only in combinations. We detected a lymphodepletion chemotherapy in six out of seven unique combinations involving TCR T cells. CAR NK cells are even rarer in clinical settings. In this approach, the CAR gene is introduced in the genome of the patient's NK cells instead of T cells. We found only two phase one trials analyzing CAR NK cells in patients with brain tumor. The first trial has an unknown status and investigated anti-mucin 1 CAR NK cells in gliomas and other cancers (NCT02839954). Another trial analyzes the intracranial administration of NK-92/5.28.z cells in HER2 positive glioblastoma. These cells represent an allogeneic NK cell population transduced with the anti-HER2-CD28 CAR gene (NCT03383978). In comparison, in other cancers (especially in hematologic malignancies), CAR NK cells seem to be more studied compared with brain tumors (Wang et al., 2020).

To date, CAR T cells and TCR T cells are one of the fastest-growing areas of in-brain cancer field. However, there are still many concerns as to whether these approaches will work out. Several strategies have been proposed to overcome challenges with CAR $\mathrm{T}$ cells in solid tumors (Rafiq et al., 2020). However, only a few of them are currently presented in clinical trials with brain tumors. Combination approaches might be helpful, but their diversity is still sparse. There is an exception, however. In our data, we found four clinical trials analyzing a promising combination of modified effector cells with checkpoint inhibitors (NCT03412877, NCT03726515, NCT04003649). We expect that many more additional CAR T-cell and TCR T-cell designs and strategies, combinations included, will enter clinical trials in the current decade.

Another increasingly developing gene therapy is the DNA vaccine class. Being both gene therapy and immune therapy, DNA vaccines are designed to provide a specific immune response against a desired antigen. The difference is the antigen used for immunization. The classic antitumor vaccines employ peptides, lysates, or vesicles, whereas DNA vaccines are DNA molecules. DNA vaccines also require a delivery method, which could be a delivery vehicle like a liposome or a technical device-an electroporator or a gene gun. Once delivered to a living cell, the DNA is supposed to be expressed into an antigen and elicit the immune response. We found that all of the trials that could be referred to DNA vaccines were initiated in 2016 and thereafter. There are four DNA vaccine approaches in five phase one brain tumor clinical trials: anti-VEGFR-2 (VMX01) (NCT02718443, NCT03750071), Wilms tumor gene-1 (WT1)/prostate-specific membrane antigen/human telomerase reverse transcriptase (hTERT) (INO-5401) (NCT03491683), and two neoantigen vaccines (NCT03988283, NCT04015700). VMX01 is an antiangiogenic vaccine against vascular endothelial growth factor receptor and is examined either alone or with avelumab (anti-PD-L1 checkpoint inhibitor). The INO-5401 vaccine is composed of three separate DNA plasmids designed against WT1 antigen, prostate-specific membrane antigen, and hTERT genes. This vaccine is a part of a combinational approach implicating interleukin (IL)-12 DNA, cemiplimab (anti-PD1 checkpoint inhibitor), and temozolomide with radiation therapy. DNA plasmids are intradermally delivered via an electroporation device (NCT03491683) (Diehl et al., 2013). The latter two vaccines should target tumorexclusive peptides, and they also require electroporation for delivery. It might be favorable to use these 
medications with other immune therapies, particularly checkpoint inhibitors, and maybe these trials will be undertaken in the future.

\section{Checkpoint Inhibitors and Immune Modulators}

1. Checkpoint Inhibitors-Checkpoints. Cancer immunotherapy is a group of approaches to treat tumors by increasing the patient's antitumor immune response rather than the direct killing of tumor cells. Checkpoint inhibitors and immune modulators (CIIMs) are an immunotherapy class that comprises agents designed to counter negative immune regulation, which is a hallmark of the tumor microenvironment as well as the agents that activate immune cells to eradicate tumor. It should be noted that we separated biologic immune modulators from small molecules with immune-modulating activities: They are included in their separate class. In brain tumor clinical trials, 31 unique drugs that belong to the CIIM were identified.

Checkpoint inhibitors [or checkpoint blockade (CB)] along with CAR T cells were called a breakthrough of the year by the Science journal in 2013 (CouzinFrankel, 2013). In 2018, James P. Allison and Tasuku Honjo were awarded The Nobel Prize in Physiology or Medicine for discovering the method of cancer immune therapy via inhibiting the immune checkpoints. The number of checkpoint inhibitors has shown a dramatic increase since 2010, with the presence of all CIIM agents in phase one trials growing from 4 to 30 at a peak in 2018. Nivolumab is a monoclonal antibody against PD1, which is expressed on $\mathrm{T}$ cells and upon activation via PD-L1/PD-L2 initiates T-cell exhaustion and apoptosis (Sharpe and Pauken, 2018). In normal conditions, this mechanism protects healthy cells from the autoimmune responses, whereas in tumor tissue, it is jeopardized and facilitates the tumor immune escape. Thus, an inhibitory property of nivolumab should benefit patients with cancer. In brain tumor trials, nivolumab has been detected in 34 combinations in 40 clinical trials in the last 10 years. This agent has been used nine times as monotherapy and 48 times in combinations. Currently, one phase two trial demonstrated no obvious clinical benefits of using nivolumab as monotherapy in patients with glioblastoma multiforme (NCT02550249). Consistently, the CheckMate 143 phase three trial showed no advantages of nivolumab monotherapy versus bevacizumab monotherapy in recurrent glioblastoma (Reardon et al., 2020). In combinations, nivolumab was applied with ipilimumab (most often), bevacizumab, temozolomide, and epacadostat as well as with other agents, including personalized antitumor vaccines and modified immune effector cells. A phase one cohort of the CheckMate 143 revealed no benefits of the nivolumab plus ipilimumab combination in recurrent glioblastoma, although the sample size is too small to make definitive conclusions. Additionally, nivolumab alone was tolerated better than in combination (Omuro et al., 2018). To date, two phase three clinical trials enrolling 1243 participants combined are studying nivolumab in combination with radiation therapy with or without temozolomide (NCT02617589, NCT02667587).

The second most investigated CB drug is pembrolizumab. This agent also targets PD1 and is presented in 19 unique combinations in 30 different clinical trials. A phase one trial of pembrolizumab revealed significant survival benefits in using neoadjuvant pembrolizumab in surgically resectable glioblastoma. Interestingly, patients who received neoadjuvant pembrolizumab besides adjuvant intervention showed almost 2-fold higher median overall survival (13.7 months) compared with the adjuvant-only arm (7.5 months) (Cloughesy et al., 2019). Pembrolizumab most frequently appeared in combinations with radiation therapy and temozolomide. It was also analyzed with abemaciclib (cyclin-dependent kinase inhibitor), bevacizumab, and several vaccines. For instance, it was combined with personalized neoantigen vaccine (NeoVax) and radiation therapy for treating glioblastoma, and this trial is currently in phase two (NCT02287428). Ipilimumab, which was frequently combined with nivolumab, is also a checkpoint inhibitor. However, in comparison with the previous two CB drugs, this agent acts on CTLA-4. CTLA-4 is a member of the CD28 subfamily of proteins. The function of CTLA- 4 is to inhibit the immune activation via competitive binding with CD80 and CD86 expressed on antigen-presenting cells (Van Coillie et al., 2020). Ipilimumab was found in 10 unique combinations in 14 clinical trials. The most common combination of the drug is with nivolumab with or without temozolomide, radiation therapy, or both. In some combinations, ipilimumab was used with bevacizumab. This combination is tolerable and demonstrated promising clinical outcomes, with $31 \%$ of patients showing a partial response (Carter et al., 2016). However, the trial was in phase one, so the sample size is small (16 patients), and the combination requires further evaluation. Currently, seven trials with ipilimumab are recruiting participants, whereas three other trials are active. An alternative to ipilimumab could be tremelimumab, which is analyzed either alone or in combination with durvalumab (anti-PD-L1 CB agent) in a phase two trial (NCT02794883).

Several other checkpoint inhibitors also target PD1, but the number of trials is small. These agents include: AMG404, cemiplimab, MGA012, pidilizumab, spartalizumab. Interestingly, AMG404 is combined with the only anti-EGFRviii/CD3 bispecific T-cell engager (AMG596) in our database (NCT03296696). Cemiplimab was analyzed in three trials, being either a monotherapy or in combination with the INO-5401 vaccine and IL- 
12 plasmid as well as with radiation therapy or with Ad-RTS-hIL-12 adenovirus encoding IL-12 (NCT03491683, NCT03690869, NCT04006119). Pidilizumab (MDV9300) was used in combination with cyclophosphamide and radiation therapy in a single trial, which demonstrated prolonged overall survival of patients with diffuse intrinsic pontine glioma (Fried et al., 2018). None of the patients received cyclophosphamide, however, despite the fact that it was mentioned in the arm description (NCT01952769). Both MGA012 and spartalizumab were investigated in combination with other checkpoint inhibitors. For example, MGA012 is a part of a combination strategy with INCAGN01876 (anti-GITR antibody) (NCT04225039), whereas spartalizumab is applied concomitantly with MBG453 (anti-Tim-3 antibody) (NCT03961971). These new drugs are expected to have CB activity; however, it has not been confirmed in the clinic yet. Another drug that is also worth noting is relatlimab, an anti-lymphocyte-activation gene $3 \mathrm{CB}$ agent that was either used alone or combined with nivolumab. Lymphocyteactivation gene 3 binds MHC class II molecules and was shown to mediate regulatory T-cell immunosuppressive function (Huang et al., 2004).

2. Checkpoint Inhibitors-Ligands. The important question in $\mathrm{CB}$ is whether to target a receptor or a ligand, and several CB drugs were designed to target the latter. One such agent is avelumab, a monoclonal antibody against PD-L1 (CD274), a ligand for PD-1. PD-L1 is expressed on various types of cells and is upregulated via cytokines, in particular, interferons. It was shown that ability of interferons to increase expression of PD-L1 might underlie the "adaptive resistance" in tumors. Namely, T cells infiltrate tumor, release proinflammatory cytokines, and thus increase expression of PD-L1, causing a negative immune regulation on themselves (Sharpe and Pauken, 2018). Avelumab might be a solution to this issue. This drug was analyzed in four unique combinations in six clinical trials with patients with brain cancer. Avelumab was also applied two times as a monotherapy. This agent was combined either with radiation therapy, axitinib, VMX01 DNA vaccine, or IDH1R132H peptide vaccine (NCT02968940, NCT03291314, NCT03750071, NCT03893903). The combination of avelumab with axitinib was well tolerated, yet it failed to meet primary efficacy endpoints in recurrent glioblastoma (Neyns et al., 2019). Another drug that targets PD-L1 is atezolizumab. In brain cancer, this drug appeared in five unique combinations in four clinical trials, and it has not been examined as monotherapy thus far. This agent is combined with radiation therapy, temozolomide (NCT03158389, NCT03174197), ipatasertib [a protein kinase Akt (AKT) inhibitor], and D2C7-IT (NCT03673787, NCT04160494). D2C7-IT is a bispecific antibody against EGFR and EGFRviii fused with immunotoxin. The combination of this agent with $\mathrm{CB}$ demonstrated promising results in preclinical glioma models (Chandramohan et al., 2019). Durvalumab is an alternative to avelumab, which also targets PD-L1. This agent was investigated in four different clinical trials, both as monotherapy and in combinations with radiation therapy, CB drugs, and olaparib.

3. Other Immune Modulators. In contrast to CBs, other immune modulators directly facilitate antitumor responses. A large portion of such agents is represented by cytokines. This is a diverse group of molecules with a broad spectrum of activities that mediate the functioning of the immune system, generation of blood cells, tissue homeostasis, and metabolism. The most abundant cytokine in our database is recombinant granulocyte macrophage colony-stimulating factor (GM-CSF) that regulates generation of monocytes, neutrophils, and dendritic cells from precursors and stem cells. It has been shown that this cytokine is sufficient to recruit dendritic cells to a vaccination site, and this is the reason for using this agent as a supplement in combinational approaches with vaccines. At high doses, however, the effect might change from immune activation to suppression (Zhan et al., 2019). The international nonproprietary name of GM-CSF is sargramostim. This agent was identified in 21 unique clinical trials and was investigated primarily in combinations with immune therapy. Most of these combinations include dendritic cells as well as peptide vaccines.

Several clinical trials examine combinations with ILs. These are also cytokines, but their function is more immune-oriented. IL-2 and IL-15 were analyzed in two trials with $\mathrm{NK}$ cells and $\mathrm{DC}$ vaccination or with NK cells alone, respectively (NCT01235845, NCT01875601). Both of the trials are relatively old and did not pass to phase two. An interesting molecule that started to appear in trials recently is NT-I7. This is a recombinant human interleukin 7 fused with the Fc region of an antibody and is expected to stimulate the proliferation of $\mathrm{T}$ cells, whereas the Fc region is used to improve the pharmacokinetics of the molecule. NT-I7 is investigated in four clinical trials either alone or in combination with radiation therapy and temozolomide (NCT02659800, NCT03619239, NCT03687957, NCT04065087). We also found combinational approaches with interferons in three clinical trials. In one trial that is recruiting participants, pegylated interferon $\alpha-2 b$ is used as monotherapy in low-grade gliomas in children (NCT02343224). The last two interesting agents of the CIIM class are fibromun and urelumab. Fibromun is an immunocytokine that acts via inducing immune response on fibronectin-expressing tumor cells. One phase one trial with fibromun monotherapy in patients with glioma is currently recruiting participants (NCT03779230). Urelumab is an agonistic monoclonal antibody that targets CD137 on immune cells to stimulate the immune 
response. In one clinical trial, it was applied either as monotherapy or combined with nivolumab to treat recurrent glioblastoma. However, according to the trial information, these arms are closed, and we found no available results on it (NCT02658981).

\section{E. Kinase Inhibitors}

The role of kinases in oncology is immense. The list of kinases that are associated with human cancers, the mutations of which are called a "driver," expands every year leading to the development of new screening approaches to study the cancer kinome (Fleuren et al., 2016). In brain tumors, the driver role is attributed to mutations in several kinases, including EGFR, fibroblast growth factor receptor (FGFR), and the phosphoinositide 3-kinase (PI3K)-AKT-mechanistic target of rapamycin (MTOR) pathway (Huse and Holland, 2010). Kinase inhibitors are small molecules that are designed to target a specific kinase or several kinases to stop tumor growth. The presence of this class was consistently high in trials during the last 10 years, yielding more than ten instances of the class in trials per year. Kinase inhibitors are the most versatile class in our database, with 91 agents investigated, and this is not including angiogenesis inhibitors, many of which are kinase inhibitors by action. Kinase inhibitors target 87 unique proteins (kinases and receptor tyrosine kinases) that account for $\sim 15 \%$ of all targets in our data. The primary and secondary targets include isoforms of several kinases: the PI3K-AKT-MTOR pathway, anaplastic lymphoma kinase, EGFR and ERBB2, FGFR, Janus kinase (JAK), KIT, cyclin-dependent kinases (CDKs), tyrosine-protein kinase Lyn, mitogen-activating protein kinase, EPHA2, tropomyosin receptor kinase, WEE1 kinase, and many other targets. The majority of agents aimed at an MTOR (14 agents), EGFR (11 agents), PI3K isoforms (6-11 agents), KIT (7 agents), anaplastic lymphoma kinase (6 agents), and FGFR isoforms (5-6 agents). Almost half of the targets are drugged by a single agent each.

From a drug perspective, the most investigated agent in the class is everolimus, an MTOR inhibitor that has been detected in 15 clinical trials. A high presence of everolimus is not surprising given that the molecule has been approved in astrocytoma and has potential application in other brain tumors. Everolimus was frequently used in combinations, including temozolomide, sorafenib (a kinase inhibitor with strong antiangiogenesis activity), lenvatinib (VEGFR inhibitor), dasatinib (BCR/ABL and SRC inhibitor), and ribociclib (CDK inhibitor). Frequently investigated kinase inhibitors also include abemaciclib, dabrafenib, nab-rapamycin, pablociclib, and many more drugs. One interesting example is nab-rapamycin. This agent is a nanoparticle albumin-bound rapamycin designed to ease the entering of rapamycin into endothelial and tumor cells. This agent has been detected in one phase two clinical trial exploring its use in five different combinations that include radiation therapy, temozolomide, lomustine, bevacizumab, and proteasome inhibitor marizomib to treat newly diagnosed glioblastoma (NCT03463265).

Despite the diversity of kinase inhibitors, to date almost all of them have not passed to the phase three trial stage yet for the treatment of brain cancer. At the moment, selumetinib, a mitogen-activating protein kinase $1 / 2$ inhibitor, was the only exception that passed to phase three trials and currently is under investigation in low-grade glioma and astrocytoma (NCT03871257, NCT04166409). Currently, this agent has been approved by the FDA to treat neurofibromatosis type I, a genetic disease associated with brain tumor development (https://www.fda.gov/news-events/ press-announcements/fda-approves-first-therapy-child ren-debilitating-anddisfiguring-rare-disease).

Kinase inhibitors are the most saturated class in neuro-oncology, given that particular targets like MTOR are drugged by 14 different agents. Despite the diversity of drugs, kinase inhibitors are rarely combined with each other. We found only 17 unique combinations wherein it contained more than one kinase inhibitor, which is $16 \%$ of the total number of unique combinations with this class. Kinase inhibitors are not frequently combined with other therapies either. In our data, 49 of 91 agents were never combined at all, and 19 were in combinations only once, thus making a median number of combinations for a kinase inhibitor being equal to zero. And this is despite the prominent phenomenon of drug resistance, which has been known since the adoption of imatinib, the first small-molecule kinase inhibitor to be approved in oncology (Cools et al., 2005; Rask-Andersen et al., 2014). One of the solutions to overcome drug resistance is to use combinational strategies. Thus, we suggest that this direction has three possible scenarios and room for improvement: The first is exploring additional kinase targets via new agents, the second is exploring new combinations of already existing agents to overcome drug resistance mechanisms, and the third is the combination of these two suggestions. It should be noted, however, that concerns, such as targeting and pharmacokinetic issues like penetration of the BBB, must be addressed as well.

\section{F. Angiogenesis Inhibitors}

Angiogenesis inhibitors have been tested in 175 trials, with the majority of trials represented by the monoclonal antibody bevacizumab being investigated 104 times. The therapeutic exploitation of the highly angiogenic nature of gliomas drove the investigation of this pharmacological class at the beginning of the decade. However, the number of phase one trials declined dramatically from 19 in 2010 to 1 by the end of 2017. Such a decline has likely resulted from the unsatisfactory clinical performance of the key agent- 
bevacizumab. A recent meta-analysis revealed that antiangiogenic therapy did not provide a significant improvement in overall survival in patients with high-grade glioma (Ameratunga et al., 2018). Moreover, the results of two randomized double blind placebo-controlled trials, (Avastin in Glioblastoma) study and (Radiation Therapy Oncology Group) 0825 study, evaluating a combination of bevacizumab with chemoradiotherapy in patients with newly diagnosed glioblastoma, demonstrated no improvement in the overall survival. In our dataset, five of six trials testing the addition of bevacizumab to standard chemoradiotherapy completed phase two but failed to progress to the subsequent phase, with two trials being terminated. Despite its approval for recurrent glioblastoma by the FDA in 2009, bevacizumab showed no substantial benefits neither alone nor in combination and has not been approved in other brain cancers thus far.

Besides the monoclonal antibody targeting VEGF, there are 27 more agents, among them 21 small molecules, 4 whole antibodies, 1 aptamer, 1 peptibody, and 1 recombinant human protein. Among the most frequent were the $\alpha_{\mathrm{v}}$-integrin inhibitor cilengtide and the multikinase inhibitors sunitinib and sorafenib. Each of these agents was tested at most in six (clingtide, sorafenib) to seven (sunitinib) trials. The other 24 agents appeared in an even smaller number of investigations. Various chemotherapeutics, including alkylating agents, topoisomerase inhibitors, antimetabolites, platinum drugs, and spindle poisons, have been investigated in combined regimens with angiogenesis inhibitors, comprising $49 \%$ of all combinations with antiangiogenic agents in our dataset. Angiogenesis inhibitors are considered to alleviate interstitial edema and favor the delivery of chemotherapeutics to the tumor tissue (Gasparini et al., 2005). However, a combination of antiangiogenic and chemotherapeutic drugs did not show sufficient superiority over chemotherapy alone because of a lack of postulated synergy between these drug classes (Ameratunga et al., 2018). The results of several studies evaluating bevacizumab with various chemotherapy agents were discouraging as well (Rinne et al., 2013). Angiogenesis inhibitors were frequently combined with additional therapies, and the median number of unique combinations for an agent is equal to two. However, only 3 out of 72 unique combinations with bevacizumab (138 for the all class) have reached phase three trials.

\section{G. Vaccines}

We previously discussed DNA vaccines that are part of gene therapy. In this section, we review other entities of the general vaccine class that belongs to immune therapy and has been one of the crucial directions of experimental brain tumor therapies for years. By its nature, a vaccine is the best therapeutic approach for simultaneously targeting multiple tumor antigens. This feature is lacking in many other popular therapeutic classes like CAR T cells, thus tumors are prone to develop antigen-escape resistance to such therapies (Majzner and Mackall, 2018). Although simultaneous targeting strategies are proposed for CAR T-cell therapy to overcome this phenomenon (Rafiq et al., 2020), vaccines are better suited for this approach. It is much more feasible to add several peptides into a vaccine composition rather than design several CAR structures. If designed properly, this class could also provide a tumor-specific treatment action, thus avoiding undesired toxicities.

The vaccine is a versatile class; in our data, we identified 67 different vaccine formulations that have been analyzed in 94 clinical trials. On average, approximately seven vaccines were in phase one clinical trials with brain tumors each year. Depending on the structure, the vaccines could be subcategorized into several subgroups: dendritic cell vaccine (36 entities), peptide (20 entities), tumor lysate (5 entities), and antibody and other structures (1 entity each). So, despite manufacturing challenges, DC-based vaccines currently dominate the field. The target spectrum of vaccines is not that versatile given that it is quite a challenge to find a suitable target in solid tumors with a reasonable safety profile and the immune response to be induced. Overall, we count 16 defined targets for vaccines in brain tumor clinical trials. The top five targets include cytomegalovirus antigens, WT1, survivin (baculoviral inhibitor of apoptosis repeat-containing 5), IDH1, and NY-ESO-1. These targets are aimed by three to five different vaccine compositions. There are also less developed targets that include EGFRviii, IL13RA2, melanoma-associated antigen 1, absent in melanoma 2, EPHA2, ERBB2, histone H3 (H3F3A), ganglioside NeuGcGM3, and tyrosinase-related protein-2. Several vaccines target more than one antigen simultaneously. For instance, one interesting vaccine design leveraged simultaneous targeting of EGFRviii and NY-ESO-1 using the attenuated bacterial cells as a delivery vehicle to dendritic cells (NCT01967758). Many of the vaccines are personalized by design, even those that did not specifically target cancer neoantigens like the Neovac vaccine (NCT03422094). Even pure lysatebased formulations or DCs stimulated with tumor lysates could be called personalized vaccines, so the number of these designs with multiple targets is quite high with 42 agents.

In contrast to kinase inhibitors, vaccines relatively frequently appear in combination with other therapies. The most common combination of vaccines is with alkylating agents and radiation therapy. Frequently, vaccines are combined with adjuvants as well as with checkpoint inhibitors and immune modulators to favor the immunogenicity. In some designs, 
vaccinations were also combined with the allogenic or autologous transfer of $\mathrm{T}$ cells to provide a better immune response. On average, each vaccine has one unique combination (median).

Despite many potential benefits, only a few vaccines have reached phase three trials. One combinational strategy analyzing DC vaccination with T-cell transplantation and stem cells is currently in a phase three trial (NCT01759810). Another study design that reached phase three was composed of DCs immunized with three components: glioblastoma stem-cell RNA, survivin, and hTERT with temozolomide therapy (NCT03548571). In another phase three trial, DCs were stimulated with tumor antigens (NCT04277221). Two trials, however, are already completed, and the outcomes are not promising. The phase three trial of the ICT-107 vaccine composed of DCs stimulated with tumor-specific peptides has been suspended because of lack of funding, which might be associated with unsatisfactory clinical performance (NCT02546102). The other trial investigated rindopepimut, a peptide vaccine targeting EGFRviii, in combination with GM-CSF and temozolomide (NCT01480479). Despite demonstrating very promising results in a phase two trial, rindopepimut showed no benefits compared with keyhole limpet hemocyanin (control peptide) in a phase three trial (Weller et al., 2017). Thus, this direction has not resulted in drug approval, yet there are still many vaccines that might be effective and are under evaluation. Given the growing popularity of checkpoint inhibitors, we expect to see additional clinical trial designs employing the novel CB drugs with vaccination.

\section{H. Metabolic Modifiers}

Metabolic modifiers approach cancer cell killing in a different manner. Given that tumor cells possess the aberrant expression of multiple proteins because of mutations, signaling pathway dysregulations, and tumor microenvironment, these cells are amenable to targeting via metabolism (Bi et al., 2020). Metabolic modifiers usually provide antitumor activities by targeting specific metabolic enzymes within cancer cells. The target spectrum is diverse and depends on a tumor type and the molecule itself. In our data, we identified 36 agents that could be referred to this drug class. The presence of metabolic modifiers in phase one trials has increased from one instance in 2010 to four instances in 2019. An interesting feature of the metabolic modifiers class is that it contains many repurposed medications. One such example is metformin, which has been detected in 10 unique combinations in 8 clinical trials. Metformin is a prominent glucose-lowering agent that has been used as first-line therapy for type two diabetes for more than 60 years in Europe. In the United States, it was approved for the same condition in 1995. This agent has been shown to inhibit the mitochondrial complex 1 to alter the cell energy status (Fujita and Inagaki, 2017). Since metformin functions in metabolic disruption as well as inhibits kinase signaling and angiogenesis, this agent has an antitumor activity (Mallik and Chowdhury, 2018). In brain tumor trials, metformin frequently appeared in combination with temozolomide and radiation therapy. In one trial, metformin was used with memantine, mefloquine, and temozolomide. This regimen was well tolerated and demonstrated promising results in newly diagnosed glioblastoma in a phase one clinical trial (Maraka et al., 2019). Metformin is also a part of an experimental metabolic regimen consisting of metformin, atorvastatin (an antihypercholesterolemic agent), doxycycline (a tetracycline-class antibiotic), and mebendazole (a spindle poison). Currently, this regimen is investigated in various types of cancer, including glioblastoma, in the phase three Safety, Tolerability, and Efficacy of Metabolic Combination Treatments on Cancer (METRICS) trial (NCT02201 381) (Agrawal et al., 2019).

The second relatively large group of metabolic modifying agents acts via targeting the IDHs. IDH enzymes catalyze the NADP + transformation of $\alpha$-ketoglutarate into isocitrate acid, which is tightly associated with the Krebs' cycle. In gliomas, these enzymes support angiogenesis, the formation of the tumor microenvironment, and invasiveness of cancer cells (Huang et al., 2019). There are two versions of IDH enzymes, IDH1 and IDH2, and both of them are targeted by agents. In our data, we found five drugs that inhibit mutated forms of IDH1: ivosidenib, vorasidenib, olutasidenib, IDH305, and DS-1001. Ivosidenib is the most common of these, being presented in four different clinical trials. It is used either as monotherapy or in combination with nivolumab, which is currently in a phase two trial (NCT04056910). Vorasidenib is an inhibitor of both mutant IDH1 and IDH2 and was identified as a monotherapy in three clinical trials. Relatively recently, a phase three trial enrolling 366 participants has been initiated to analyze the effectiveness of vorasidenib in IDH-mutated gliomas (NCT04164901). The other three agents targeting IDH1 were in phase one trials. IDH2 is less targeted than IDH1, and we found only two agents that act on mutated IDH2: vorasidenib and enasidenib. Enasidenib was analyzed to treat advanced solid tumors, including gliomas, in a single phase one/two trial (NCT02273739). The trial was completed in 2016, but no results have been published since.

Another prominent group of metabolic modifiers is the inhibitors of IDO1. IDO1 is a member of the L-tryptophan kynurenine metabolic pathway and catalyzes the rate-limiting step of converting L-tryptophan into 
$N$-formylkynurenine. In cancer, this pathway has multiple roles in tumor immune suppression, cancer cell mobility, and metastasis (Platten et al., 2019). IDO1 is targeted by four agents: indoximod, BMS-986205, PF06840003, and epacadostat. Indoximod is a methylated tryptophan and the most tested of these four agents that appeared in five unique combinations in three clinical trials. The most investigated combination of indoximod is with temozolomide. PF-06840003 was studied in a single trial, but it was terminated because of a sponsor decision (NCT02764151). Epacadostat and BMS986205 are in phase one trials and combined with nivolumab with or without temozolomide and radiation. One trial with epacadostat has been recently completed (NCT02327078).

Other metabolic modifiers were much less frequent and mostly stay at the phase one in single trials, with a few exemptions. One is the previously mentioned phase three Safety, Tolerability and Efficacy of Metabolic Combination Treatments on Cancer (METRICS) trial, wherein atorvastatin is combined with metformin, doxycycline, and mebendazole (NCT02201381, NCT02796261). Another phase three trial investigates a combination of eflornithine with lomustine. Eflornithine is an irreversible inhibitor of ornithine decarboxylase that is required for polyamine biosynthesis. Polyamines possess diverse functions within tumor cells and are linked with oncogenic signaling pathways, including the phosphatase and tensin homolog-PI3K-mTOR, WNT signaling, and RAS pathways (Casero et al., 2018).

\section{Transcription Modifiers: Tumor Targeting at a Different Level}

Most of the targeted therapies act on receptors or on the pathway members that transduce the signal from a receptor, ultimately leading to the expression of target proteins. Usually, the last chain in the pathway is represented by a transcription factor that upon biochemical modification becomes activated and translocates into the nucleus to initiate the expression of target genes. A portion of targeted therapies acts on transcription factors or associated proteins rather than other pathway members. An interesting trend is an increase in the number of transcription modifiers in clinical trials, which rose from one agent per year in 2010 up to three agents per year in 2019, with 21 agents cumulatively identified to date. Several of these agents could be subclassified into four groups: E3 ubiquitin-protein ligase $\mathrm{Mdm}(\mathrm{MDM}) 2$ inhibitors, bromodomain and extraterminal motif (BET) protein family inhibitors, JAK/signal transducer and activator of transcription (STAT) axis inhibitors, and hypoxia-inducible factor $2 \alpha$ (HIF2A) inhibitors. MDM2 is an E3 ubiquitin-ligase whose primary function is to inhibit the activity of the key tumor suppressor gene TP53. MDM2 is deregulated and overexpressed in various tumors, including glioblastoma (Wade et al., 2013). Three agents that target MDM2 were identified: the peptide ALRN-6924 that inhibits both MDM2 and MDM4, the small molecular inhibitor idasanutlin that blocks the interaction between P53 and MDM2, and AMG 232 that demonstrate similar characteristics to idasanutlin. Each of the three drugs is in phase one clinical trials (NCT03654716, NCT03158389, NCT01723020, NCT03107780). Azurin-derived cell-penetrating peptide p28 exhibits a close action to MDM2 inhibitors. However, in contrast to the previous group, p28 binds directly to P53 to protect it from degradation (Yamada et al., 2013). This agent was well tolerated yet failed to demonstrate promising outcomes in pediatric patients with recurrent or progressive central nervous system tumors in a phase one trial (Lulla et al., 2016).

$\mathrm{BET}$ proteins are epigenetic regulators that contain tandem bromodomains (BD1 and BD2) as well as extraterminal and C-terminal domains. The primary function of these proteins is the regulation of the expression activity of various transcription factors and oncogenes, including nuclear factor $-\kappa \mathrm{B}$, Myc proto-oncogene family, E2F2, CDK6, and others. Thus, there is a rationale for using BET inhibitors for cancer therapy (Stathis and Bertoni, 2018). Four BET inhibitors were identified in trials for brain cancer: birabresib, INCB057643, BMS-986158, and CC-90010. However, the actual clinical performance of BET inhibitors in neuro-oncology is questionable. A phase two trial analyzing birabresib (MK-8628) in recurrent glioblastoma was terminated because of a lack of activity of the investigative drug (NCT02296476). In contrast, INCB057643 failed a phase one/two clinical trial in advanced solid tumors because of safety reasons rather than activity (NCT02711137). The other two inhibitors BMS-986158 and CC-90010 are still under examination in recently started phase one trials, so there are not any results yet (NCT03936465, NCT04047303).

Inhibitors of JAK/STAT and HIF2A are not frequent in brain tumor clinical trials, with each of these groups containing only two agents. JAK/STAT is a proliferative signaling pathway that consists of JAK, which in turn activates STAT proteins. Upon activation by phosphorylation, STAT proteins dimerize and translocate into the nucleus to activate expression of target genes (O'Shea et al., 2015). WP1066 and napabucasin, JAK/STAT inhibitors, were investigated in high-grade gliomas in phase one clinical trials (NCT01904123, NCT02315534). However, despite years passed, these agents have not reached phase two. HIF2A is also a transcription factor and is induced under hypoxic conditions common for tumors. It has been discovered that this protein is crucial for glioblastoma stem cells (the most aggressive and proliferating cells in a tumor) and predominantly 
expressed in this population and also correlates with poor patient survival ( $\mathrm{Li}$ et al., 2009). Two monotherapies, PT2977 and PT2385, that target HIF2A were identified in our dataset. PT2977 is in phase one, whereas PT2385 has completed the phase two trial (NCT02974738, NCT03216499).

Selinexor is an interesting drug in which the transcription modifying action is based on targeting nuclear transport rather than transcription factors. This molecule inhibits exportin, a protein found on the nuclear membrane, which exports various tumor suppressor transcription factors from the nucleus, therefore hampering their antiproliferative activity and facilitating tumor progression. Selinexor in combination with dexamethasone received accelerated approval in the United States for the treatment of myeloma in 2019 (Syed, 2019). For the treatment of brain tumors, this agent has appeared in four clinical trials. Early trials of selinexor analyzed this agent as monotherapy (NCT01607905, NCT01986348, NCT02323880), whereas a recent trial initiated in 2020 is analyzing this drug in combination with temozolomide (NCT04216329). Despite the fact that the first two trials had been completed, we did not find results.

\section{J. Oncolytic Viruses Are Still in Early Development}

Oncolytic viruses are a relatively unique direction for cancer therapy. Oncolytic viruses are defined by their ability to selectively replicate in tumor cells without damaging the normal ones. To date, viruses are designed using either genetic engineering employing immune-modulating and safety modifications or using natural tumor-tropic viruses, such as reovirus (Fukuhara et al., 2016). In 2010, there were no trials analyzing oncolytic viruses in brain tumors; in 2017, this number peaked, reaching three trials per year. We identified 10 unique oncolytic viruses in brain tumor clinical trials. These viruses originated from different viral taxons: Four viruses are derived from herpes simplex virus (HSV); two were adenoviruses; and measles virus, parvovirus, polio virus, and reovirus have only one entity of their origin. Most of the viruses were used as monotherapies. Oncolytic adenovirus AD5-DNX-2401 is the most frequent member of its class and appeared in six clinical trials. This agent was also combined with interferon $\gamma$-1b, temozolomide, or pembrolizumab. Combinations with interferon or pembrolizumab might be promising therapeutic designs because of the ability of oncolytic viruses to elicit the antitumor immune response (Fukuhara et al., 2016), which could be enhanced by the immune-modulating activity of interferon $\gamma-1 b$ and pembrolizumab. Currently, a combination of AD5-DNX-2401 with pembrolizumab is one of a few combinations that has reached phase two trials on different types of brain tumors (NCT02798406). Another relatively abundant virus is PVS-RIPO, a modified polio virus, which was analyzed in three clinical trials and reached a phase two stage to be investigated in patients with high-grade glioma (NCT02986178). To date, only the two abovementioned viruses have reached phase two clinical trials.

\section{K. PARP Inhibitors: Now beyond Breast Cancer}

PARP inhibitors are small molecules that are designed to inhibit the enzyme PARP. PARP is a crucial enzyme participating in the DNA base excision repair, homologous recombination, and nonhomologous end-joining processes that are crucial for cell homeostasis. PARP inhibitors demonstrated impressive clinical performance in BRCA1/2 mutated tumors, and their application seems to expand beyond these cancers (Tangutoori et al., 2015). In our data, we identified seven unique PARP inhibitors that were analyzed in 19 clinical trials. Of these seven agents, olaparib, pamiparib, and veliparib were the most common. Olaparib was found in eight clinical trials. If combined, this drug was used with temozolomide with or without radiation as well as with cediranib or durvalumab. Pamiparib and veliparib were used in three clinical trials each. To date, PARP inhibitors mostly are in the phase two stage. However, veliparib is currently under evaluation with or without temozolomide in glioblastoma/gliosarcoma patients in a phase two/three trial (NCT02152982).

\section{Radiopharmaceuticals: A Targeted Radiation Therapy}

Another interesting class is represented by radiopharmaceutical agents. This is a type of radiation therapy that is drug-based, compared with standard radiation therapy. Despite being relatively rare, this class has demonstrated increasing interest, especially in the last 3 years. We count eight unique agents identified as radiopharmaceuticals. This class could be subcategorized, depending on the structure: one group is radiolabeled antibodies, and another is radiolabeled small molecules or peptides. In our database, two drugs are small molecules, four drugs are radioantibody conjugate, two are peptides, and one agent is a macromolecular complex (liposomal rhenium Re186). In the majority of trials, radiopharmaceuticals appeared as monotherapies. Currently, many agents have not progressed beyond the phase one clinical trial stage. An exception is yttrium Y 90-DOTA-tyr3-octreotide, a small radiolabeled peptide that has reached phase two clinical trials and currently is under evaluation in several tumors, including medulloblastoma (NCT02441088, NCT03273712). Also, lutetium Lu 177 dotatate, a radiolabeled peptide, is tested in two phase two trials on inoperable/progressive meningioma (NCT03971461, NCT04082520). 


\section{Embryonic Pathway Inhibitors}

Embryonic pathway inhibitors represent a specific branch of targeted therapies designed to target the oncogenic pathways that are predominantly active during the embryonal development. These pathways include Hedgehog, Notch, and Wnt signaling. These pathways also are commonly attributed to the concept of cancer stem cells and may play a significant role in tumor development (Takebe et al., 2015). We identified seven agents that function as embryonic pathway inhibitors. Depending on the target, these agents could be subdivided into two groups: $\gamma$-secretase inhibitors (Notch signaling) (RO4929097, MK-0752) and smoothened (SMO) inhibitors (Hedgehog signaling) (LEQ506, sonidegib, vismodegib, glasdegib, ZSP1602). All of these seven drugs are small molecules. In several trials, these agents were combined with kinase inhibitors, angiogenesis inhibitors, alkylating agents, and radiation. The number of agents has declined sharply from eight molecules in 2010 to one in 2018 in phase one trials.

One might ask the reason for such a decline, and probably this is related to the overall uncertainties and limitations in the cancer stem-cell concept (Pastrana et al., 2011; Rahman et al., 2011; Medema, 2013; Tarasov et al., 2019b). Currently, none of the agents in the class reached phase three trials. Consistently, the percentage of discontinued trials (terminated, suspended, withdrawn) for embryonic pathway inhibitors is one of the largest among all therapeutic classes (Supplemental Fig. 1). However, there are six clinical trials that are still recruiting participants and may successfully demonstrate the rationale in using embryonic pathway inhibitors for brain cancer therapy.

\section{N. Histone Deacetylase Inhibitors: Reaching the Third Stage}

HDACs represent a family of regulatory enzymes that control the functioning of various cellular processes through the modification of gene expression. HDACs function via removing the acetyl groups from $\mathrm{N}$-acetyl lysine in histones. Upon deacetylation, histones bind with DNA, thus preventing its transcription. Generally, there are 18 HDACs that demonstrate distinct expression patterns and perform slightly different functions. For further information, read these publications (Medema, 2013; Ho et al., 2020; Verza et al., 2020). Nine HDAC inhibitors were identified in trials for neuro-oncology. The most investigated agent is vorinostat that has been tested in 10 trials, which is followed by valproic acid (six trials) and panobinostat (four trials). HDAC inhibitors were analyzed both alone and in combinational regimens, which included temozolomide, radiation therapy, and, rarely, bevacizumab or another agent. Of all agents, only vorinostat and valproic acid have reached phase two/three and phase three clinical trials, respectively, and are under evaluation in combinations in several types of brain tumors (NCT03243461, NCT01236560, NCT02265770). Vorinostat was analyzed in combination with temozolomide and bevacizumab, whereas valproic acid was combined with either temozolomide or another chemotherapeutic agent. An interesting example of HDAC inhibitors is tinostamustine, a fusion molecule composed of an alkylating agent bendamustine and vorinostat. To date, this agent is under evaluation with radiation therapy in a phase one trial (NCT03452930). MTX110 is a water-soluble panobinostat administered as a nano formulation through a convectionenhanced delivery system. Loading a drug into water-soluble nanoparticles allows to effectively use the convection-enhanced delivery system to bypass the BBB and avoid systemic toxicity in comparison with the poorly soluble oral formulation, which failed to penetrate the BBB (Singleton et al., 2018). In our dataset, the earliest trials of panobinostat analyzed this agent without a delivery system.

Despite two drugs that have demonstrated promising performances, the overall number of agents in phase one trials declined from five in 2010 to one in 2019. This class exhibits the fourth largest percentage of discontinued clinical trials among all therapeutic classes. It should be noted, however, that the terminated trials are relatively old, and only one trial, which analyzed the combination of vorinostat, erlotinib, and temozolomide, has failed because of issues with treatment (toxicity) (NCT01110876). Thus, this downward tendency might be a data artifact, taking into account the small number of all trials in total (27) and a small number of trials per year (5 or fewer).

\section{Target Exploration and the Drug-Target Network}

Drugs frequently share different targets, thus generating complex interaction networks. Given the spatial complexity of the data, the only way to visualize such interactions is to use the drug-target network as it has been done by our group in the previous publication (Rask-Andersen et al., 2011). The concept of the drug-target network is based on a graph theory, wherein each node represents a drug or a target, whereas edges show the act of targeting between a drug and its corresponding target. The concept of the drug-target network was introduced by Yildirim et al. (2007), in which authors used it to analyze the interactions among FDA-approved drugs and investigational agents from the drug bank database. For our analysis, we used drugs that we found in the clinical trials on brain tumors since 2010. To build the 


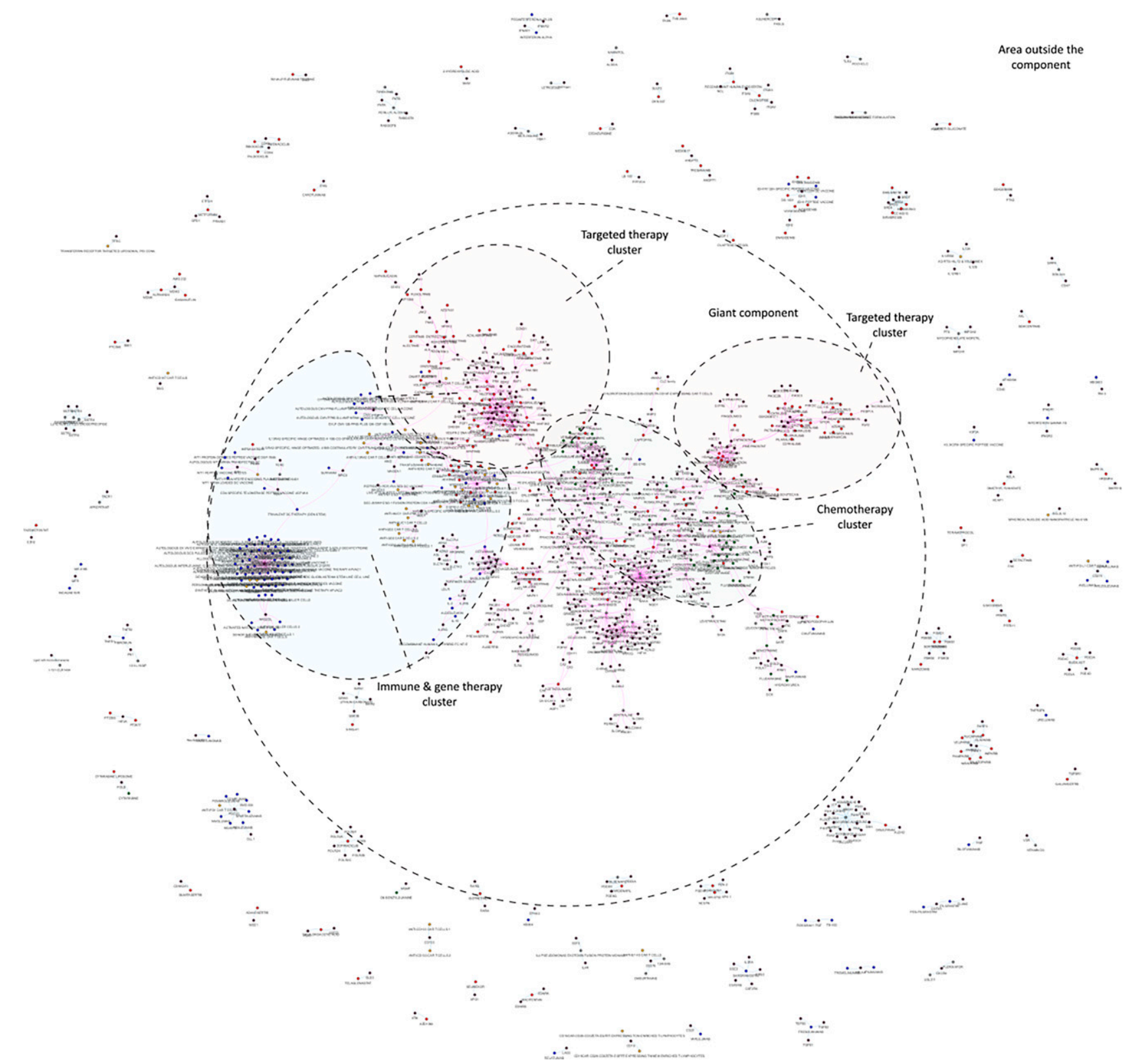

Fig. 6. Drug-target network. The drug-target network and interactions. The area in the center with magenta edges is the giant component. The giant component is divided into several superclusters. Colors of the nodes indicate the following: red is targeted therapy, green is chemotherapy, blue is immune therapy, yellow is gene therapy, gray is other therapies, and vinous is target. This network has been created via $\mathrm{R}$ programming language. Data are to February 2020

network we used 518 agents and 581 targets. The interactive version of this network in the HTML script can be found in additional supplementary materials.

Similar to the previous publications (Yildirim et al., 2007; Rask-Andersen et al., 2011), the network was assembled with the giant component in the center (Fig. 6). The giant component is the largest subgraph within a given graph. The component itself is selfclusterized according to the pharmacological action of a particular molecule, although it slightly differs compared with our classification. The network demonstrates the following large clusters in the giant component: chemotherapy, thalidomide derivatives, metabolic modifiers, kinase inhibitors, PI3K-MTOR inhibitors, topoisomerase inhibitors, HDAC inhibitors, and biotech class composed of drugs and targets for vaccines and other cell therapies. The therapeutic entities in the giant component could be considered more established since there is more information on these agents, and they share the target spectrum at least to some extent. Currently, $72 \%$ of the agents (375) and $69 \%$ of all targets (402) are a part of the giant component. Agents outside the component could be called relatively unique given they do not share the targets with conventional therapies. We assume that these agents might have the potential to deliver more unexpected (both positive and negative) results 
compared with those inside the largest subgraph in the network since it is harder to predict the outcome of a therapy if it does not possess similar targets with previously analyzed therapies. In our network, these small components include IDH-targeting agents, PD1-targeting agents, PD-L1-targeting agents, CDK inhibitors, $\gamma$-secretase inhibitors, integrin inhibitors, PARP inhibitors, proteasome inhibitors, bromodomain protein family inhibitors, and other drugs. Interestingly, in terms of drug classes, transcriptions modifiers and CIIMs demonstrated mostly peripheral localization. Namely, only 4 of 21 agents belonging to transcription modifiers and only 5 of 31 CIIMs were presented in the giant component.

Our next step was to gather aggregated data on each target and analyze how these targets are explored in clinical trials. For the first analysis, we specifically used targets for small molecules and antibodies. We did not include targets that are antigens for vaccines or other advanced biologicals like cell therapies and gene therapies since these treatments are not inhibiting targets and rather employ them to selectively eliminate tumors based on the target expression. Thus, antibodies arguably could be included in both analyses because of their ambivalent nature. To augment the data on targets, we calculated the following characteristics for each target in our dataset: the presence score, the maximal phase for a target, the number of trials in which this target has been exploited, the number of unique agents drugging a target, and the quantitative trend. Table 2 illustrates the top 20 targets for brain tumors in the clinical trials (an extended version is in Supplemental Table 2). Logically, the most studied targets are DNA and VEGFA since they both have at least one agent that has been approved by the FDA. PD1 (PDCD1) is the third most analyzed target because of the growing interest toward checkpoint inhibitors. Many kinases and RTKs, including kinase insert domain receptor, EGFR, FLT1, KIT, and MTOR, were actively studied and drugged by several agents. EGFR is an especially notable target. This protein, including mutated or regular forms, has been drugged by 25 unique agents (without cell therapies and vaccines) either as a primary or a secondary target. During the last 10 years, these interventions have been and are being explored in 49 clinical trials, of which none have passed to the phase three stage thus far. Such a high presence of this target is not surprising given that this protein is involved in many proliferative signaling pathways and is amplified in about $40 \%$ of patients with glioblastoma and is believed to predict response to kinase inhibitors (Ludwig and Kornblum, 2017). However, the clinical data provide unsatisfactory results, showing a lack of a durable response to the treatment that in some cases was worse compared with temozolomide
(Patel et al., 2012). A recently published meta-analysis showed almost no benefits in the overall survival of patients with glioblastoma when using anti-EGFR therapies (Lee et al., 2020). Such discouraging results for one of the most promising targets question its validity and urge for additional exploration of novel targets and approaches. According to our analysis, 45 primary and secondary targets for nonadvanced biologicals and 5 targets for advanced biologicals (vaccines, cell therapies, and gene therapies) have entered clinical trials during 2018 and 2019.

The pool of drug targets for vaccines and cellular and gene therapies is sparse compared with those of small molecules and antibodies. Overall, we count 40 different targets, which mostly are cell-surface antigens of tumor cells (Supplemental Fig. 2). No agents have been approved yet, and the actual number of defined targets reaching the phase three ultimate clinical trial stage is only six (EGFR, ERBB2, IL13RA2, melanoma-associated antigen 1, absent in melanoma 2, tyrosinase-related protein-2) (Supplemental Table 3). The pool of new targets that entered trials in 2020 at a date of writing is also small and contains targets for CHLOROTOXIN (EQ)-CD28-CD3ל-CD19Texpressing CAR T cells (chloride channels, annexin A2, and matrix metalloproteinase-2).

Many of the targets both for advanced biologicals and small molecules are in the earliest stages of exploration. Twenty targets of advanced biologic agents $(\sim 48 \%)$ are exploited by a single agent only. Eighteen targets $(\sim 46 \%)$ have not progressed further than phase one trials. To compare, $290(\sim 51 \%)$ out of 530 targets for small molecules and antibodies also are drugged by single agents. However, only 172 $(\sim 32 \%)$ out of 545 targets are in the phase one stage, suggesting that targets for advanced biologicals are clinically underexplored comparing with more regular drugs.

\section{Trends in Combinations}

Combination therapies represent one of the key strategies in the modern and classic pharmacology, which, if designed properly, could potentiate the effect of individual medications, thus providing substantial clinical benefits for a patient. In oncology, combinational strategies are proposed to overcome drug resistance by creating multiple bottlenecks in cancer cell survival or to limit the toxicities of chemotherapy.

In brain tumor trials, we identified 557 unique combinations after the manual characterization of all treatment arms in 981 clinical trials included in the analysis. Using our pharmacological classification, we calculated the mean and the median number of unique combinations per each agent of each class (Fig. 7A). This analysis revealed that the area of combinations is mostly studied for specific drug classes, 
TABLE 2

Trends in most common drug targets

This table illustrates the top 20 targets for drugs other than vaccines and advanced biologicals, like CAR T cells. Note, proteins binding with the Fc region of antibodies have been removed from the table. The presence is calculated by the number of trials in which a particular target has been

drugged. If a single trial contains more than one agent that targets a particular drug target, this trial is counted as the number of these agents. Max phase column illustrates the maximal phase of a clinical trial in which a particular target has appeared. Trials with phase four have not been included because of the fact they contain agents that were never approved for brain tumors, and likely their phase is not equal for all analyzed treatment arms. Approved drug targets are labeled as approved. The number of clinical trials is calculated by the number of trials in which a particular target has been drugged, and each trial is counted only once, no matter the number of agents against the target. The number of unique drugs illustrates the number of drugs that exploit the target. For each drug, both pivotal and secondary targets were taken into account. The trend was calculated as follows: if a 3-year avg. of the target presence during 2017, 2018, and 2019 is $10 \%$ or more higher than the avg. during the 10-year period, the trend is upward, whereas if it is $10 \%$ or more below than the avg. during the 10-year period, the trend is downward. The year column illustrates the first year the target appeared during the analyzed 10 years of brain cancer trials. Data are to February 2020.

\begin{tabular}{|c|c|c|c|c|c|c|}
\hline Target & Presence & Max. Phase & Number of Trials with Target & Number of Unique Agents Drugging the Target & Trend & Earliest Year \\
\hline DNA & 422 & Approved & 370 & 27 & No trend & 2010 \\
\hline VEGFA & 109 & Approved & 108 & 3 & Downward trend & 2010 \\
\hline PDCD1 & 78 & 3 & 78 & 7 & Upward trend & 2013 \\
\hline NR1I2 & 64 & 3 & 62 & 14 & No trend & 2010 \\
\hline KDR & 60 & 2 & 57 & 19 & No trend & 2010 \\
\hline PTGS2 & 59 & 3 & 54 & 9 & No trend & 2010 \\
\hline PPARG & 52 & 3 & 51 & 6 & Upward trend & 2010 \\
\hline EGFR & 50 & 2 & 49 & 25 & No trend & 2010 \\
\hline MTOR & 48 & Approved & 47 & 14 & Downward trend & 2010 \\
\hline PTGS1 & 46 & 3 & 46 & 5 & Upward trend & 2010 \\
\hline TUBB1 & 46 & 3 & 45 & 16 & Downward trend & 2010 \\
\hline ACAT1 & 45 & 3 & 45 & 4 & Upward trend & 2010 \\
\hline FLT1 & 45 & 2 & 45 & 13 & No trend & 2010 \\
\hline KIT & 45 & 2 & 42 & 13 & Downward trend & 2010 \\
\hline PDGFRB & 42 & 2 & 39 & 11 & Downward trend & 2010 \\
\hline FLT4 & 41 & 2 & 41 & 12 & Downward trend & 2010 \\
\hline HRH2 & 40 & 3 & 40 & 2 & Upward trend & 2010 \\
\hline TOP1 & 36 & 2 & 36 & $\overline{7}$ & Downward trend & 2010 \\
\hline PDGFRA & 34 & 2 & 33 & 12 & Downward trend & 2010 \\
\hline TOP1MT & 33 & 2 & 33 & 4 & Downward trend & 2010 \\
\hline TOP2A & 32 & 3 & 29 & 11 & Downward trend & 2010 \\
\hline HDAC2 & 28 & 3 & 28 & 10 & Downward trend & 2010 \\
\hline FGFR1 & 26 & 2 & 25 & 10 & Downward trend & 2010 \\
\hline TACSTD2 & 26 & 2 & 26 & 4 & Downward trend & 2010 \\
\hline TYMS & 26 & 2 & 23 & 7 & Downward trend & 2010 \\
\hline
\end{tabular}

DNA, deoxyribonucleic acid; EGFR, epidermal growth factor receptor; FGFR1, fibroblast Growth Factor Receptor 1; FLT1, vascular endothelial growth factor receptor 1;

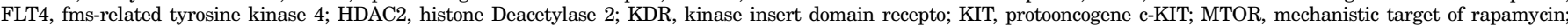

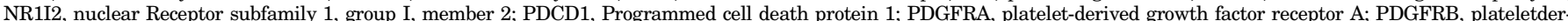

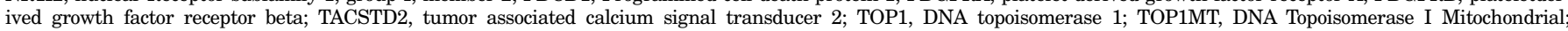
TOP2A, DNA Topoisomerase II Alpha; TUBB1, tubulin beta-1 chain; TYMS, thymidylate synthetase; VEGFA, vascular endothelial growth factor A.

overlooking several pharmacological directions like kinase inhibitors and other types of targeted and immune therapies. Currently, more than two-thirds of all therapeutic classes have the median number of combinations per agent being equal to one or less. Strikingly, this number is equal to zero combinations for kinase inhibitors, the most iconic direction for targeted therapy. On the other hand, chemotherapy, proteasome inhibitors, angiogenesis inhibitors, and immune modulators were found to be the most actively combined directions.

The significant difference between the mean value and the median value for combinations indicates the extremes in the data, suggesting that combinational designs are skewed not only toward specific pharmacological classes but also toward individual drugs. To analyze and visualize the dependencies of combinations in the data, we introduce the concept of a combination network that links close combinations together based on the presence of a subcombination within a combination, using the algorithm depicted in Fig. 7B. Generally, this algorithm finds whether one combination is a part of another combination and links them if it is. The interactive version of the network can be found in additional supplementary materials; the high-resolution image is in Supplemental Fig. 3. This network visualizes the prevailing patterns in the tested combinations in clinical trials.

Similar to the drug-target network, the combination network assembles with the giant component in its center. Interestingly, the network is clusterized based on the major pharmacological direction and corresponding therapy within a combination, which includes chemotherapy, angiogenesis inhibitors, and immune therapy. The network also demonstrates that most of the combinations are based and centered around temozolomide with or without radiation therapy, bevacizumab, nivolumab, pembrolizumab, poly ICLC, cyclophosphamide, carboplatin, and lomustine. This uneven grouping of combination designs suggests that combinations in clinical trials are predominantly designed with a small and limited number of agents and agent combination patterns. This tendency is logical, considering that almost half of combination designs in the giant component centered 
A

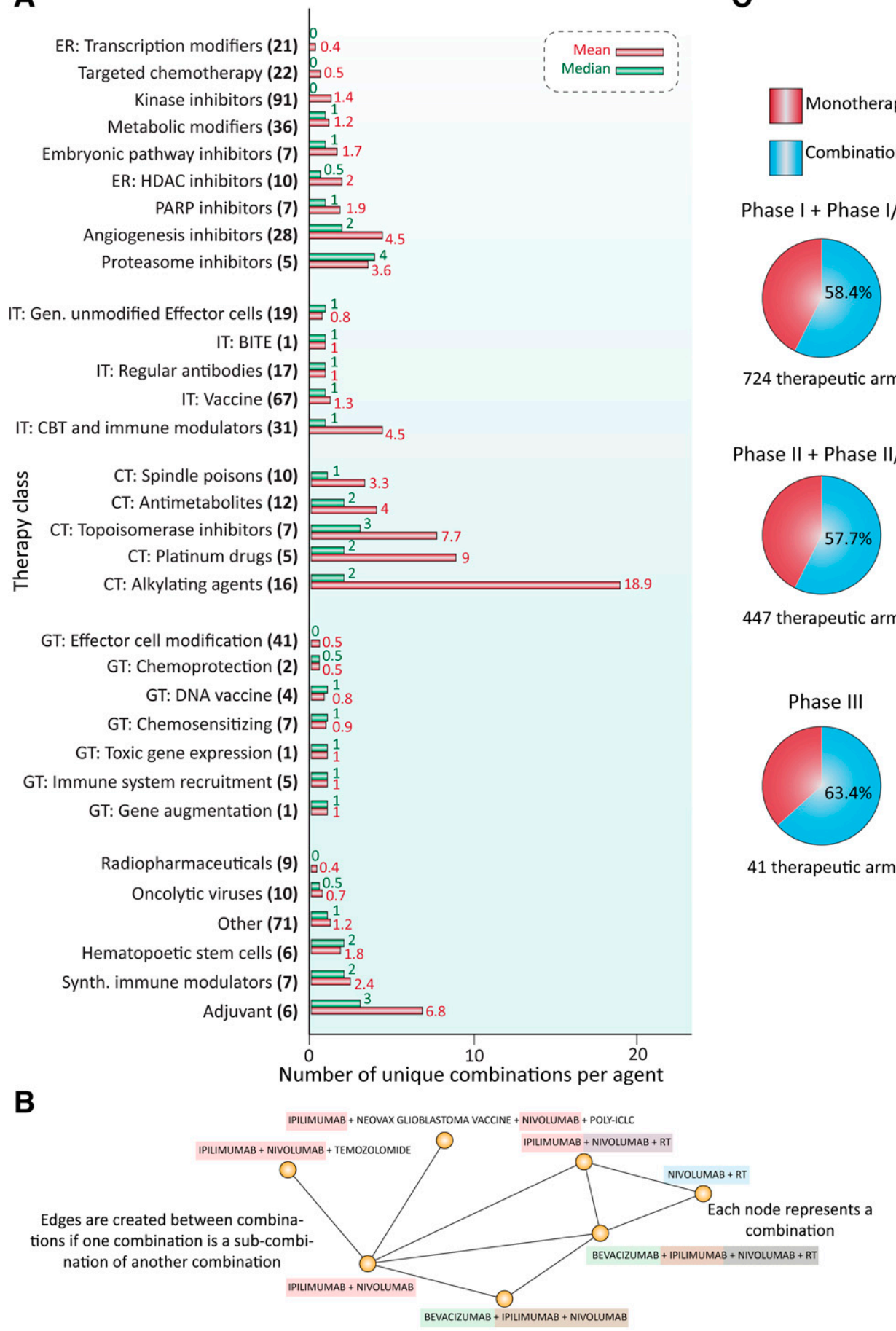

C

Fig. 7. Combination analysis. (A) Number of unique combinations per therapeutic class. Each bar corresponds to the median (green color) or the mean number (red color) of unique combinations for each drug in a particular therapeutic class. Bold numbers in parentheses are the number of agents in each corresponding class. The large difference among means and medians indicates that combinational designs are skewed toward particular drugs. For instance, in alkylating agents, this difference is related to the fact that temozolomide has been detected in 209 unique combinations (more than $30 \%$ of all designs). (B) Schematic representation of network algorithm. The schematic representation of the algorithm forming a combination network from the input vector of all combinations. Each node corresponds to the unique combination. If one combination is a subcombination of another combination, the algorithm creates an edge among two nodes. (C) The percentage of combinational and monotherapy arms among clinical trials of different phases. The number below each pie chart shows the number of treatment arms. This analysis is quantitative and does not remove the same combination designs from different clinical trials. By the term "genetically unmodified effector cells" we mean cells with unmodified immune effector function. BITE, bispecific T-cell engager; CBT, checkpoint blockade therapy; CT, chemotherapy; ER, expression regulators; Gen, genetically; GT, gene therapy; IT, immune therapy; Synth., synthetic. around radiation therapy and temozolomide that are the standard-of-care therapy. As expected, the giant component does not show a cluster for kinase inhibitors, and many members of that class both alone and in combination are located outside of it. The additional version of the network that simply links drugs together if they were found in combination and is not limited to design patterns shows the very close picture. Similarly, drugs are distributed around a limited pool of agents (Supplemental Fig. 4).
To investigate the pharmacological similarities in designed combinations and monotherapies, we performed a quantitative analysis of treatment arms (without removing similar arms from different trials) presented in the data. This analysis showed the most common monotherapies and combinations in clinical trials. As expected, the top of arm designs relies on several types of monotherapies, including alkylating agents, CAR T and TCR T cells, kinase inhibitors, vaccines, and angiogenesis inhibitors, whereas the 
most common combination is radiation therapy with temozolomide. Surprisingly, kinase inhibitors were relatively frequently presented in the top 50 treatment arms. This could indicate that although individual agents among kinase inhibitors are rarely combined and the diversity of combinations is small, the whole direction is investigated with combinations demonstrating repetitive patterns. The graphical representation of this analysis could be found in supplementary figures (Supplemental Figs. 5 and 6).

Some treatment arms might be more intriguing and promising compared with others. Over the years, various research groups have proposed different combination strategies for rational cancer therapy. Such combinations could demonstrate more potent tumor killing, reduce toxicity, facilitate antitumor immunity, or overcome drug resistance. Having the data on treatment arms, we decided to explore how the proposed solutions are aligned with the actual practice in the clinical trials on brain tumors and how often these strategies are implemented. To do so, we manually searched publications on combination strategies for brain tumors and other cancers to identify combinational designs and then checked whether these designs are presented in our database. The summary of this analysis is provided in Table 3. As it can be seen, previously proposed therapy designs, which include combinations with TTF, checkpoint inhibitors, CAR $\mathrm{T}$ cells, and vaccines, are indeed presented among clinical trials. However, the majority of combinational trials are at the recruiting stage and in early phases, whereas several designs with advanced biologicals were not tested in brain tumors. Moreover, the number of such therapeutic arms is relatively small, indicating avenues for further investigation.

\section{Discussion and Future Perspective}

The high number and diversity of agents within clinical trials illustrate a major transformation within cancer therapy research. The pool of agents in clinical trials is highly diversified and involves different types of drug structures that leverage several distinct pharmacological approaches. Our structural classification suggests at least 42 technology-based classes that include 2 small-molecule platforms, 6 antibody platforms, 15 cell platforms, 5 protein platforms, 8 viral platforms, and 6 other drug structures. In terms of pharmacology, we can highlight five major classes of therapy: targeted therapies, immune therapies, chemotherapy, gene therapy, and other therapies that in total comprise 32 specific treatment directions. The recent increasing interest in cell therapies has made them the third largest experimental therapeutic approach for cancer therapy.

Consistently, the large pool of pharmacological agents has resulted in the wide diversity of drugged targets in brain cancers. Currently, enzymes, especially kinases, and receptors represent the first priority both for targeted and immune therapies. Miscellaneous proteins, transporters, and ligands share a relatively small fraction of the target pool. However, a strong focus on checkpoint inhibitors will likely lead to the growth of cell-surface ligand targets since it is a promising alternative to classic checkpoints. Interestingly, soluble ligands for checkpoints may be an additional promising direction that aligns with the trend for soluble ligand targeting (Asanuma et al., 2020; Attwood et al., 2020). Besides ligands, we expect to see additional targets among kinases and metabolic enzymes as well as additional antigen targets for advanced biologicals. Our target analysis, however, indicates a declining rate in the target adoption for small molecules and antibodies: On average 20 new targets per year have entered clinical trials during 2017-2019, which is 3-fold lower than those in 2011-2013 (Supplemental Fig. 7). New unique targets are thus appearing at a relatively modest rate.

Our analysis shows four classes, alkylating agents, checkpoint inhibitors and immune modulators, modified effector cells, and metabolic modifiers, that show large growth (Fig. 8). There is an increasing number of trials per year for these classes. Several agents, including anti-PD-1 checkpoint inhibitors, have been found outside the giant component in our network, which suggests that these approaches are relatively novel for brain tumors. The especially strong impetus for modified immune cells and CIIM agents could be directly seen in the figure. Furthermore, shifts in the immune and gene therapies are not only quantitative but qualitative as well. For instance, checkpoint inhibitors are embracing many strategies and are beyond targeting PD1 and CTLA-4 than a few years ago. The key concern of the class relates to the biologic nature of these therapies. Given that CB drugs are antibodies, it is challenging to provide robust delivery of these medications into the brain. CAR cells account for 17 different targeting approaches alone without taking into account various mutated versions of targets. Additionally, $(\gamma \delta) \mathrm{T}$ cells that are both the unique population of $\mathrm{T}$ cells and an emerging class of T-cell therapy have been also found in clinical trials for brain cancer (NCT04165941) (Sebestyen et al., 2020). It is interesting that new approaches for CAR T-cell engineering, which include CRISPRengineered $\mathrm{T}$ cells and macrophage-based CAR cells, are actively explored in preclinical and clinical stages in many other cancers (Yu et al., 2020). Likely, these approaches will be investigated soon for brain tumors.

There is also interesting new development for transcription and metabolic modifiers. Interestingly, several agents, like metformin, are being repurposed. 


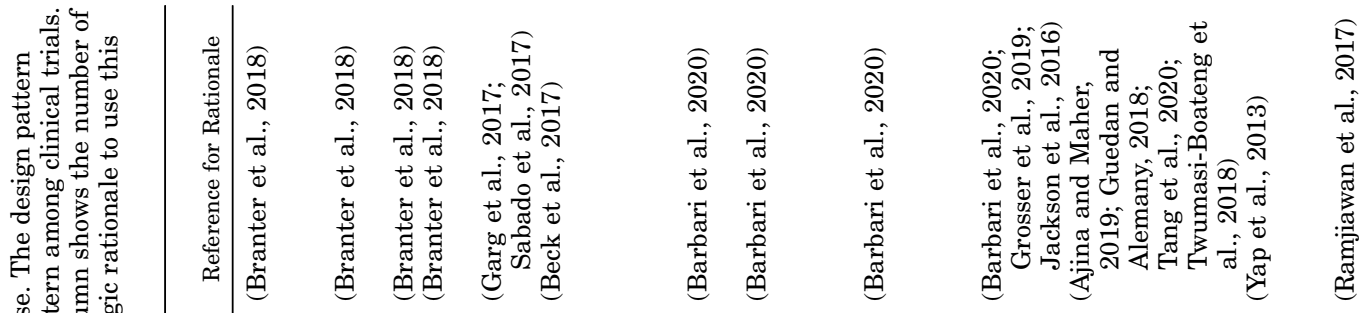

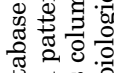

蛋.

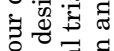

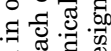

용

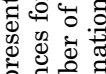

ब

o 8 ¿

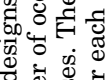

它这

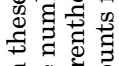

ฐ ఫ

की

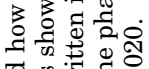

चี

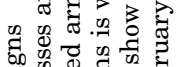

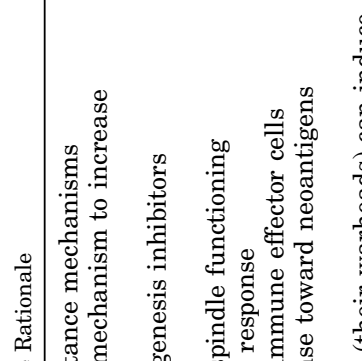

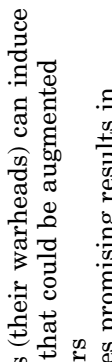

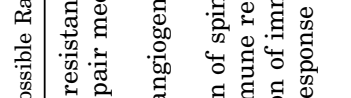

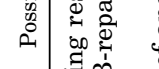

:

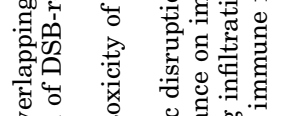

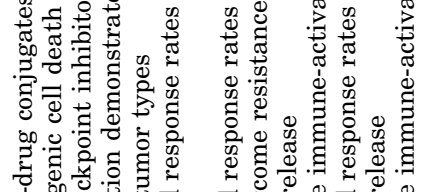

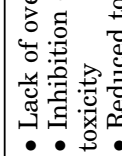

.

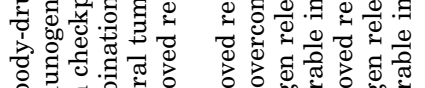

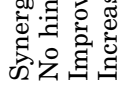

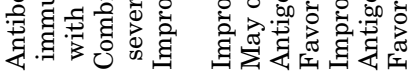

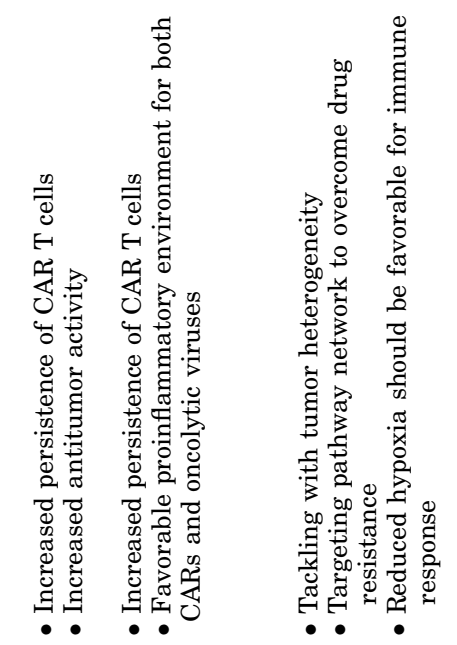

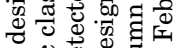

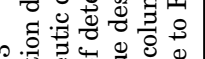

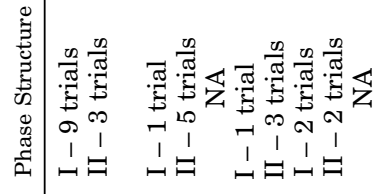

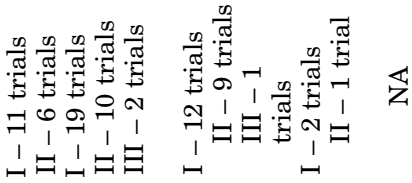

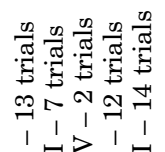

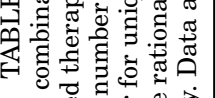

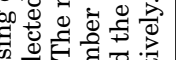

揢

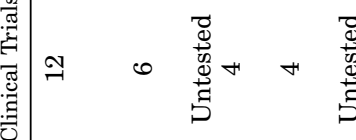

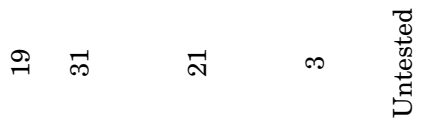

ส กั

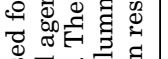

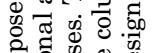

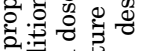

बี

o. 해

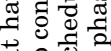

焉

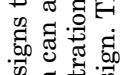

क्षे

๔

. हैं

:

है है

of o웡

뭉

割

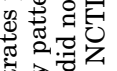

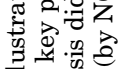

年

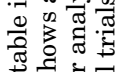

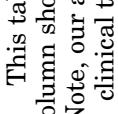

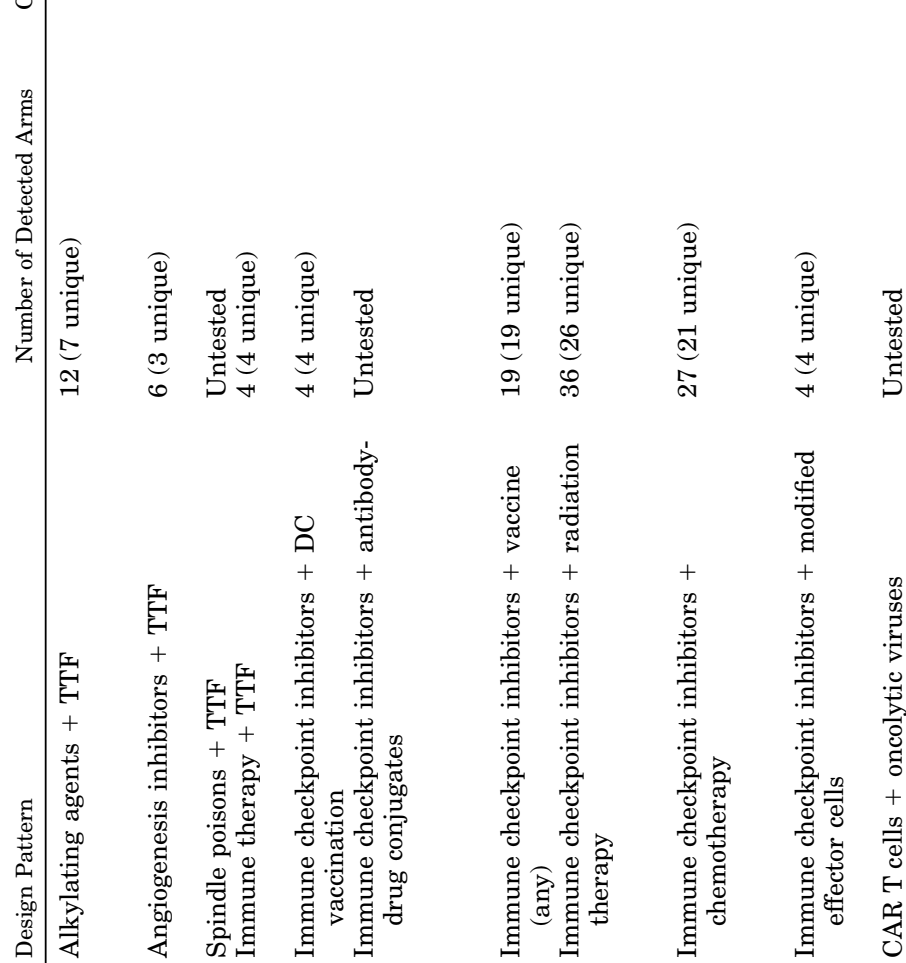

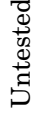

เコュル

$\Xi$
0
0
0
00
0
0
0
0
0
0
0
0
0
0
0

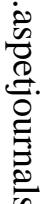

$\stackrel{9}{00}$

$\stackrel{2}{=}$

超

㞼

일

? 

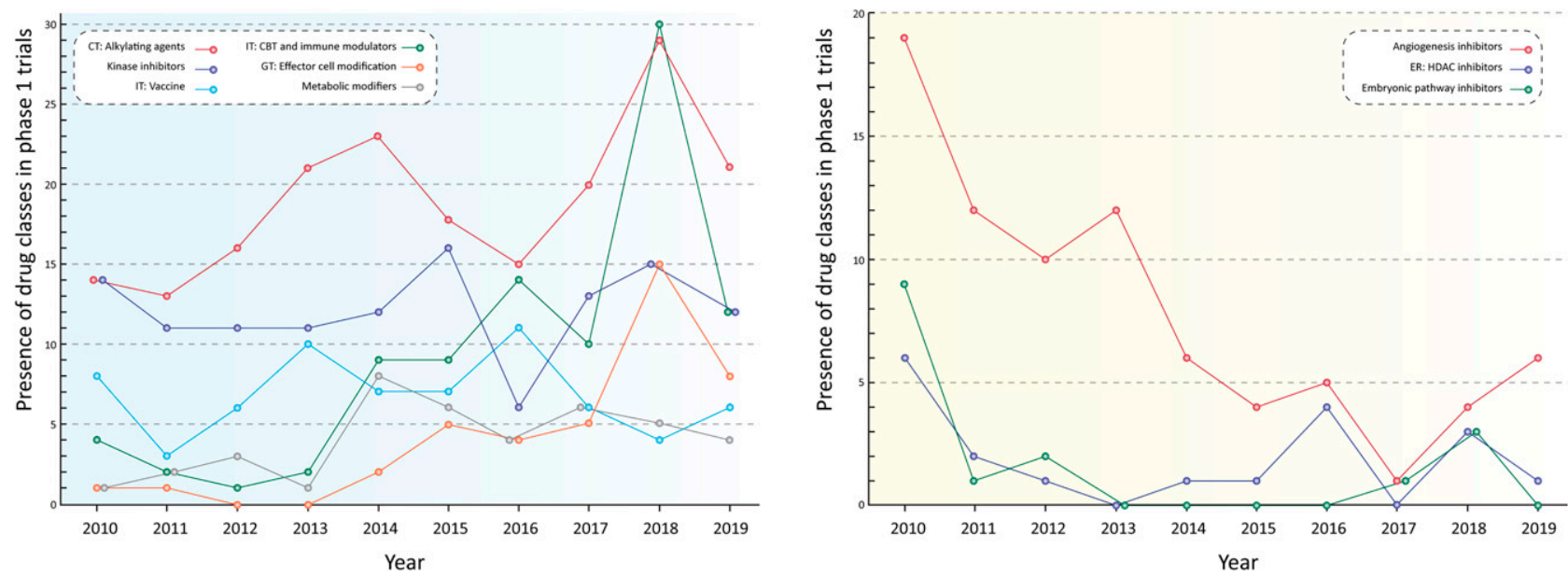

Fig. 8. Quantitative trends for phase one clinical trials. Trends in phase one clinical trials. This figure illustrates the trends for the most significant classes in phase one clinical trials of the dataset. The presence was calculated using the combined number of all drug entities of a particular class per year. If a particular drug appeared in a clinical trial, it was counted only once, no matter the number of arms with this agent in a trial. If a particular drug was analyzed in several different clinical trials, each of the trials was counted. If several agents of the same class appeared in the same trial, it is counted as the number of these agents. The colors of trend lines correspond to pharmacological classes. Data are to December 2019. CBT, checkpoint blockade therapy; CT, chemotherapy; ER, expression regulator; GT, gene therapy; IT, immune therapy.

For instance, at the time of writing, the antipsychotic agent chlorpromazine is tested in combination with radiation therapy and temozolomide to treat glioblastoma multiforme in a phase two trial (NCT04224441). This is the second agent after ONC201 (selective dopamine receptor D2/dopamine receptor D3 antagonist) to analyze targets related to dopamine receptor signaling for the purpose of treating glioblastoma. Interestingly, the opposite approach is investigated as well. For instance, previously approved dopamine signaling agonists, including cabergoline, bromocriptine, and ropinirole, are analyzed as a therapy of pituitary tumors (including nonfunctioning specimens) (NCT01 620138, NCT03038308). Moreover, other classes like oncolytic viruses, PARP inhibitors, and DNA vaccines are also demonstrating increasing presence in clinical trials (Fig. 8; Supplemental Fig. 8).

Surprisingly, we see no growth in terms of number of trials per year for two very important classes: the kinase inhibitors and vaccines. Currently, only five vaccine candidates reached phase three clinical trials, whereas for kinase inhibitors, only one candidate is at this stage. Notably, angiogenesis inhibitors, HDAC inhibitors, and embryonic pathway inhibitors showed the declining presence in phase one clinical trials, and it is possible that this decline is primarily specific for brain cancers. This trend could change, however, as during the last 3 years the angiogenesis inhibitors (mostly bevacizumab) show an increasing number of studies in combination with checkpoint inhibitors, and this might be a promising combination that can prompt interest in other combinations with this class. Additionally, HDAC inhibitors are also being actively developed in other cancers. For instance, five agents have been approved for hematologic malignancies, and 27 more agents are under investigation in various clinical trials (unpublished data).

Our analysis of treatment arms demonstrates a diversity of combinations. However, many innovative therapies that include kinase inhibitors, CAR T cells, vaccines, and targeted therapies are frequently tested as single agents, whereas combinations are predominantly designed for well established therapies like alkylating chemotherapy. For instance, the median number of combinations per agent for antimetabolites and alkylating agents is two, whereas it is zero for kinase inhibitors and modified effector cells. Interestingly, we also see a clear preference for individual drugs. For example, temozolomide alone has been detected in 209 unique combinations. This tendency can be seen in the combination networks in which multiple edges are directed toward the key therapies, such as temozolomide. Currently, many treatment designs rely on combining previously approved therapies, at least in other cancers. There seem to be large untapped opportunities in treatment strategies that could be composed of agents that might not work as monotherapies. Interestingly, there are existing cases of such a strategy in cancer therapy. For example, the FDA approved a combination of two kinase inhibitors (encorafenib with binimetinib for the treatment of BRAF V600E/K mutant melanoma in 2018), and both of these drugs were not indicated for use as monotherapies and had not been previously authorized (binimetinib, encorafenib). Moreover, the concept of combinational therapy has been proven to be successful in some other types of therapies undergoing intense developmental periods, such as in HIV, wherein three agents are typically used concomitantly to contain the virus (Deeks et al., 2013; Atta et al., 
2019). This study also provides evidence that the use of combinations seems to favor clinical translation. We see that the percentage of combinational therapies is almost 6\% higher in the trials of phase three compared with those in phases one and two (Fig. 7C) in the analyzed dataset. Thus, designing rational combinations could be an important objective for future studies.

\section{Authorship Contributions}

Participated in research design: Sokolov, Dostdar, Attwood, Chubarev, Tarasov, Schiöth.

Contributed new reagents or analytic tools: Sokolov, Dostdar, Attwood, Chubarev, Tarasov, Schiöth.

Performed data analysis: Sokolov, Dostdar, Krasilnikova, Ilina,

Nabieva, Lisitsyna.

Wrote or contributed to the writing of the manuscript: Sokolov, Dostdar, Attwood, Schiöth.

\section{References}

Agrawal S, Vamadevan P, Mazibuko N, Bannister R, Swery R, Wilson S, and Edwards S (2019) A new method for ethical and efficient evidence generation for off-label medication use in oncology (a case study in glioblastoma). Front Pharmacol 10:681.

Aijaz A, Li M, Smith D, Khong D, LeBlon C, Fenton OS, Olabisi RM, Libutti S, Tischfield J, Maus MV, et al. (2018) Biomanufacturing for clinically advanced cell therapies. Nat Biomed Eng 2:362-376.

Ajina A and Maher J (2019) Synergistic combination of oncolytic virotherapy with CAR T-cell therapy. Prog Mol Biol Transl Sci 164:217-292.

Ameratunga M, Pavlakis N, Wheeler H, Grant R, Simes J, and Khasraw M (2018) Anti-angiogenic therapy for high-grade glioma. Cochrane Database Syst Rev 11:CD008218.

An Z, Aksoy O, Zheng T, Fan QW, and Weiss WA (2018) Epidermal growth factor receptor and EGFRvIII in glioblastoma: signaling pathways and targeted therapies. Oncogene 37:1561-1575.

Asanuma K, Nakamura T, Hayashi A, Okamoto T, Ino T, Asanuma Y, Hagi T, Kita K, Nakamura K, and Sudo A (2020) Soluble programmed death-ligand 1 rather than PD-L1 on tumor cells effectively predicts metastasis and prognosis in soft tissue sarcomas. Sci Rep 10:9077.

Atta MG, De Seigneux S, and Lucas GM (2019) Clinical pharmacology in HIV therapy. Clin J Am Soc Nephrol 14:435-444.

Attwood MM, Jonsson J, Rask-Andersen M, and Schiöth HB (2020) Soluble ligands as drug targets. Nat Rev Drug Discov 19:695-710.

Attwood MM, Rask-Andersen M, and Schiöth HB (2018) Orphan drugs and their impact on pharmaceutical development. Trends Pharmacol Sci 39:525-535.

Baeuerle PA and Reinhardt C (2009) Bispecific T-cell engaging antibodies for cancer therapy. Cancer Res 69:4941-4944.

Balza E, Carnemolla B, Mortara L, Castellani P, Soncini D, Accolla RS, and Borsi L (2010) Therapy-induced antitumor vaccination in neuroblastomas by the combined targeting of IL-2 and TNFalpha. Int J Cancer 127:101-110.

Bao X, Pastan I, Bigner DD, and Chandramohan V (2016) EGFR/EGFRvIIItargeted immunotoxin therapy for the treatment of glioblastomas via convectionenhanced delivery. Receptors Clin Investig 3:e1430.

Barbari C, Fontaine T, Parajuli P, Lamichhane N, Jakubski S, Lamichhane P, and Deshmukh RR (2020) Immunotherapies and combination strategies for immunooncology. Int J Mol Sci 21:5009.

Barnholtz-Sloan JS, Ostrom QT, and Cote D (2018) Epidemiology of brain tumors Neurol Clin 36:395-419.

Beck A, Goetsch L, Dumontet C, and Corvaia N (2017) Strategies and challenges for the next generation of antibody-drug conjugates. Nat Rev Drug Discov 16:315-337.

Bexell D, Svensson A, and Bengzon J (2013) Stem cell-based therapy for malignant glioma. Cancer Treat Rev 39:358-365.

Bi J, Chowdhry S, Wu S, Zhang W, Masui K, and Mischel PS (2020) Altered cellular metabolism in gliomas - an emerging landscape of actionable codependency targets. Nat Rev Cancer 20:57-70.

Bourgeois M, Bailly C, Frindel M, Guerard F, Chérel M, Faivre-Chauvet A, Kraeber-Bodéré F, and Bodet-Milin C (2017) Radioimmunoconjugates for treating cancer: recent advances and current opportunities. Expert Opin Biol Ther 17:813-819.

Boyd AW, Bartlett PF, and Lackmann M (2014) Therapeutic targeting of EPH receptors and their ligands. Nat Rev Drug Discov 13:39-62.

Branter J, Basu S, and Smith S (2018) Tumour treating fields in a combinational therapeutic approach. Oncotarget 9:36631-36644.

Bretscher C and Marchini A (2019) H-1 parvovirus as a cancer-killing agent: past, present, and future. Viruses 11:562.

Burger MC, Zhang C, Harter PN, Romanski A, Strassheimer F, Senft C, Tonn T, Steinbach JP, and Wels WS (2019) CAR-engineered NK Cells for the treatment of glioblastoma: turning innate effectors into precision tools for cancer immunotherapy. Front Immunol 10:2683.
Carter T, Shaw H, Cohn-Brown D, Chester K, and Mulholland P (2016) Ipilimumab and bevacizumab in glioblastoma. Clin Oncol (R Coll Radiol) 28:622-626.

Casero Jr RA, Murray Stewart T, and Pegg AE (2018) Polyamine metabolism and cancer: treatments, challenges and opportunities. Nat Rev Cancer 18:681-695.

Cavaco M, Castanho MARB, and Neves V (2017) Peptibodies: An elegant solution for a long-standing problem. Biopolymers [published ahead of print].

Cesarini V, Scopa C, Silvestris DA, Scafidi A, Petrera V, Del Baldo G, and Gallo A (2020) Aptamer-based in vivo therapeutic targeting of glioblastoma. Molecules 25:4267.

Chandramohan V, Bao X, Yu X, Parker S, McDowall C, Yu YR, Healy P, Desjardins A, Gunn MD, Gromeier M, et al. (2019) Improved efficacy against malignant brain tumors with EGFRwt/EGFRvIII targeting immunotoxin and checkpoint inhibitor combinations. J Immunother Cancer 7:142.

Chandran SS and Klebanoff CA (2019) T cell receptor-based cancer immunotherapy: Emerging efficacy and pathways of resistance. Immunol Rev 290:127-147.

Chen D, Sha H, Hu T, Dong S, Zhang J, Liu S, Cao H, Ma R, Wu Y, Jing C, et al. (2018) Cytokine-induced killer cells as a feasible adoptive immunotherapy for the treatment of lung cancer. Cell Death Dis 9:366.

Claus EB, Walsh KM, Wiencke JK, Molinaro AM, Wiemels JL, Schildkraut JM, Bondy ML, Berger M, Jenkins R, and Wrensch M (2015) Survival and low-grade glioma: the emergence of genetic information. Neurosurg Focus 38:E6

Cloughesy TF, Mochizuki AY, Orpilla JR, Hugo W, Lee AH, Davidson TB, Wang AC, Ellingson BM, Rytlewski JA, Sanders CM, et al. (2019) Neoadjuvant antiPD-1 immunotherapy promotes a survival benefit with intratumoral and systemic immune responses in recurrent glioblastoma. Nat Med 25:477-486.

Cools J, Maertens C, and Marynen P (2005) Resistance to tyrosine kinase inhibitors: calling on extra forces. Drug Resist Updat 8:119-129.

Couzin-Frankel J (2013) Breakthrough of the year 2013. Cancer immunotherapy. Science 342:1432-1433

D'Ippolito E, Schober K, Nauerth M, and Busch DH (2019) T cell engineering for adoptive $\mathrm{T}$ cell therapy: safety and receptor avidity. Cancer Immunol Immunother 68:1701-1712.

Deeks SG, Lewin SR, and Havlir DV (2013) The end of AIDS: HIV infection as a chronic disease. Lancet 382:1525-1533.

Derissen EJ, Beijnen JH, and Schellens JH (2013) Concise drug review: azacitidine and decitabine. Oncologist 18:619-624.

Dhodapkar MV, Sznol M, Zhao B, Wang D, Carvajal RD, Keohan ML, Chuang E, Sanborn RE, Lutzky J, Powderly J, et al. (2014) Induction of antigen-specific immunity with a vaccine targeting NY-ESO-1 to the dendritic cell receptor DEC205. Sci Transl Med 6:232ra51.

Diehl MC, Lee JC, Daniels SE, Tebas P, Khan AS, Giffear M, Sardesai NY, and Bagarazzi ML (2013) Tolerability of intramuscular and intradermal delivery by CELLECTRA $(\circledR)$ adaptive constant current electroporation device in healthy volunteers. Hum Vaccin Immunother 9:2246-2252.

DiMasi JA, Reichert JM, Feldman L, and Malins A (2013) Clinical approval success rates for investigational cancer drugs. Clin Pharmacol Ther 94:329-335.

Fang F, Xiao W, and Tian Z (2017) NK cell-based immunotherapy for cancer. Semin Immunol 31:37-54

Federici L, Capelle L, Annereau M, Bielle F, Willekens C, Dehais C, LaigleDonadey F, Hoang-Xuan K, Delattre JY, Idbaih A, et al. (2020) 5-Azacitidine in patients with IDH1/2-mutant recurrent glioma. Neuro Oncol 22:1226-1228.

Ferguson FM and Gray NS (2018) Kinase inhibitors: the road ahead. Nat Rev Drug Discov 17:353-377.

Fitzmaurice C, Akinyemiju TF, Al Lami FH, Alam T, Alizadeh-Navaei R, Allen C, Alsharif U, Alvis-Guzman N, Amini E, Anderson BO, et al. (2018) Global, regional, and national cancer incidence, mortality, years of life lost, years lived with disability, and disability-adjusted life-years for 29 cancer groups, 1990 to 2016: a systematic analysis for the global burden of disease study. JAMA Oncol 4:1553-1568.

Fleuren ED, Zhang L, Wu J, and Daly RJ (2016) The kinome 'at large' in cancer. Nat Rev Cancer 16:83-98.

Foreman PM, Friedman GK, Cassady KA, and Markert JM (2017) Oncolytic virotherapy for the treatment of malignant glioma. Neurotherapeutics 14:333-344.

Fried I, Lossos A, Ben Ami T, Dvir R, Toledano H, Ben Arush MW, Postovski S, Abu Kuidar A, Yalon M, Weintraub M, et al. (2018) Preliminary results of immune modulating antibody MDV9300 (pidilizumab) treatment in children with diffuse intrinsic pontine glioma. J Neurooncol 136:189-195.

Fujita Y and Inagaki N (2017) Metformin: new preparations and nonglycemic benefits. Curr Diab Rep 17:5.

Fukuhara H, Ino Y, and Todo T (2016) Oncolytic virus therapy: a new era of cancer treatment at dawn. Cancer Sci 107:1373-1379.

Gambardella V, Tarazona N, Cejalvo JM, Lombardi P, Huerta M, Roselló S, Fleitas T, Roda D, and Cervantes A (2020) Personalized medicine: recent progress in cancer therapy. Cancers (Basel) 12:1009.

Garg AD, Coulie PG, Van den Eynde BJ, and Agostinis P (2017) Integrating nextgeneration dendritic cell vaccines into the current cancer immunotherapy landscape. Trends Immunol 38:577-593.

Gasparini G, Longo R, Fanelli M, and Teicher BA (2005) Combination of antiangiogenic therapy with other anticancer therapies: results, challenges, and open questions. J Clin Oncol 23:1295-1311.

González FE, Gleisner A, Falcón-Beas F, Osorio F, López MN, and Salazar-Onfray F (2014) Tumor cell lysates as immunogenic sources for cancer vaccine design. Hum Vaccin Immunother 10:3261-3269.

Gromeier M and Nair SK (2018) Recombinant poliovirus for cancer immunotherapy. Annu Rev Med 69:289-299.

Grosser R, Cherkassky L, Chintala N, and Adusumilli PS (2019) Combination immunotherapy with CAR T cells and checkpoint blockade for the treatment of solid tumors. Cancer Cell 36:471-482. 
Guedan S and Alemany R (2018) CAR-T cells and oncolytic viruses: joining forces to overcome the solid tumor challenge. Front Immunol 9:2460.

Hajeri PB, Sharma NS, and Yamamoto M (2020) Oncolytic adenoviruses: strategies for improved targeting and specificity. Cancers (Basel) 12:1504.

Harshyne LA, Hooper KM, Andrews EG, Nasca BJ, Kenyon LC, Andrews DW, and Hooper DC (2015) Glioblastoma exosomes and IGF-1R/AS-ODN are immunogenic stimuli in a translational research immunotherapy paradigm. Cancer Immunol Immunother 64:299-309.

Hauser AS, Attwood MM, Rask-Andersen M, Schiöth HB, and Gloriam DE (2017) Trends in GPCR drug discovery: new agents, targets and indications. Nat Rev Drug Discov 16:829-842.

Ho TCS, Chan AHY, and Ganesan A (2020) Thirty years of HDAC inhibitors: 2020 hindsight. J Med Chem 63:12460-12484.

Hodgins JJ, Khan ST, Park MM, Auer RC, and Ardolino M (2019) Killers 2.0: NK cell therapies at the forefront of cancer control. J Clin Invest 129:3499-3510.

Hos BJ, Tondini E, van Kasteren SI, and Ossendorp F (2018) Approaches to improve chemically defined synthetic peptide vaccines. Front Immunol 9:884.

Huang CT, Workman CJ, Flies D, Pan X, Marson AL, Zhou G, Hipkiss EL, Ravi S, Kowalski J, Levitsky HI, et al. (2004) Role of LAG-3 in regulatory T cells. Immunity 21:503-513.

Huang J, Yu J, Tu L, Huang N, Li H, and Luo Y (2019) Isocitrate dehydrogenase mutations in glioma: from basic discovery to therapeutics development. Front Oncol 9:506.

Huse JT and Holland EC (2010) Targeting brain cancer: advances in the molecular pathology of malignant glioma and medulloblastoma. Nat Rev Cancer 10:319-331.

Hyman DM, Taylor BS, and Baselga J (2017) Implementing genome-driven oncology. Cell 168:584-599.

Jackson HJ, Rafiq S, and Brentjens RJ (2016) Driving CAR T-cells forward. Nat Rev Clin Oncol 13:370-383.

Kawakami M, Kawakami K, and Puri RK (2003) Interleukin-4-Pseudomonas exotoxin chimeric fusion protein for malignant glioma therapy. $J$ Neurooncol 65:15-25.

Konkankit VV, Kim W, Koya RC, Eskin A, Dam MA, Nelson S, Ribas A, Liau LM, and Prins RM (2011) Decitabine immunosensitizes human gliomas to NY-ESO-1 specific $\mathrm{T}$ lymphocyte targeting through the Fas/Fas ligand pathway. $J$ Transl Med 9:192

Kumar V, Kumar V, McGuire T, Coulter DW, Sharp JG, and Mahato RI (2017) Challenges and recent advances in medulloblastoma therapy. Trends Pharmacol Sci 38:1061-1084.

Labrijn AF, Janmaat ML, Reichert JM, and Parren PWHI (2019) Bispecific antibodies: a mechanistic review of the pipeline. Nat Rev Drug Discov 18:585-608.

Lee A, Arasaratnam M, Chan DLH, Khasraw M, Howell VM, and Wheeler H (2020) Anti-epidermal growth factor receptor therapy for glioblastoma in adults. Cochrane Database Syst Rev 5:CD013238.

Levine BL, Miskin J, Wonnacott K, and Keir C (2016) Global manufacturing of CAR T cell therapy. Mol Ther Methods Clin Dev 4:92-101.

Li Z, Bao S, Wu Q, Wang H, Eyler C, Sathornsumetee S, Shi Q, Cao Y, Lathia J, McLendon RE, et al. (2009) Hypoxia-inducible factors regulate tumorigenic capacity of glioma stem cells. Cancer Cell 15:501-513.

Lin A, Giuliano CJ, Palladino A, John KM, Abramowicz C, Yuan ML, Sausville EL, Lukow DA, Liu L, Chait AR, et al. (2019) Off-target toxicity is a common mechanism of action of cancer drugs undergoing clinical trials. Sci Transl Med 11:eaaw8412.

Lopes A, Vandermeulen G, and Preat V (2019) Cancer DNA vaccines: current preclinical and clinical developments and future perspectives. Journal of experimental \& clinical cancer research. CR (East Lansing Mich) 38:146.

Ludwig K and Kornblum HI (2017) Molecular markers in glioma. J Neurooncol 134:505-512.

Lulla RR, Goldman S, Yamada T, Beattie CW, Bressler L, Pacini M, Pollack IF, Fisher PG, Packer RJ, Dunkel IJ, et al. (2016) Phase I trial of p28 (NSC745104), a non-HDM2-mediated peptide inhibitor of p53 ubiquitination in pediatric patients with recurrent or progressive central nervous system tumors: a pediatric brain tumor consortium study. Neuro-oncol 18:1319-1325.

Lum LG and Thakur A (2011) Targeting T cells with bispecific antibodies for cancer therapy. BioDrugs 25:365-379.

Macarron R, Banks MN, Bojanic D, Burns DJ, Cirovic DA, Garyantes T, Green DV, Hertzberg RP, Janzen WP, Paslay JW, et al. (2011) Impact of high-throughput screening in biomedical research. Nat Rev Drug Discov 10:188-195.

Mahmood I and Green MD (2005) Pharmacokinetic and pharmacodynamic considerations in the development of therapeutic proteins. Clin Pharmacokinet 44:331-347.

Majzner RG and Mackall CL (2018) Tumor antigen escape from CAR T-cell therapy. Cancer Discov 8:1219-1226.

Mallik R and Chowdhury TA (2018) Metformin in cancer. Diabetes Res Clin Pract 143:409-419.

Maraka S, Groves MD, Mammoser AG, Melguizo-Gavilanes I, Conrad CA, Tremont-Lukats IW, Loghin ME, O'Brien BJ, Puduvalli VK, Sulman EP, et al. (2019) Phase 1 lead-in to a phase 2 factorial study of temozolomide plus memantine, mefloquine, and metformin as postradiation adjuvant therapy for newly diagnosed glioblastoma. Cancer 125:424-433.

Marenco-Hillembrand L, Wijesekera O, Suarez-Meade P, Mampre D, Jackson C, Peterson J, Trifiletti D, Hammack J, Ortiz K, Lesser E, et al. (2020) Trends in glioblastoma: outcomes over time and type of intervention: a systematic evidence based analysis. J Neurooncol 147:297-307.

McNeill KA (2016) Epidemiology of Brain Tumors. Neurol Clin 34:981-998.

Medema JP (2013) Cancer stem cells: the challenges ahead. Nat Cell Biol 15:338-344.
Met Ö, Jensen KM, Chamberlain CA, Donia M, and Svane IM (2019) Principles of adoptive T cell therapy in cancer. Semin Immunopathol 41:49-58.

Mount NM, Ward SJ, Kefalas P, and Hyllner J (2015) Cell-based therapy technology classifications and translational challenges. Philos Trans $R$ Soc Lond B Biol Sci 370:20150017.

Msaouel P, Dispenzieri A, and Galanis E (2009) Clinical testing of engineered oncolytic measles virus strains in the treatment of cancer: an overview. Curr Opin Mol Ther 11:43-53.

Munos B (2009) Lessons from 60 years of pharmaceutical innovation. Nat Rev Drug Discov 8:959-968.

Nair S and Dhodapkar MV (2017) Natural killer T cells in cancer immunotherapy. Front Immunol 8:1178.

Neyns B, Salama LB, Awada G, Cremer JD, Schwarze JK, Seynaeve L, Four SD, Fischbuch L, Vanbinst A-M, Everaert H, et al. (2019) GLIAVAX: a stratified phase II clinical trial of avelumab and axitinib in patients with recurrent glioblastoma. J Clin Oncol 37:2034-2034

O'Shea JJ, Schwartz DM, Villarino AV, Gadina M, McInnes IB, and Laurence A (2015) The JAK-STAT pathway: impact on human disease and therapeutic intervention. Annu Rev Med 66:311-328.

Olin MR, Pluhar GE, Andersen BM, Shaver R, Waldron NN, and Moertel CL (2014) Victory and defeat in the induction of a therapeutic response through vaccine therapy for human and canine brain tumors: a review of the state of the art. Crit Rev Immunol 34:399-432.

Omuro A, Vlahovic G, Lim M, Sahebjam S, Baehring J, Cloughesy T, Voloschin A, Ramkissoon SH, Ligon KL, Latek R, et al. (2018) Nivolumab with or without ipilimumab in patients with recurrent glioblastoma: results from exploratory phase I cohorts of CheckMate 143. Neuro-oncol 20:674-686.

Ovacik M and Lin K (2018) Tutorial on monoclonal antibody pharmacokinetics and its considerations in early development. Clin Transl Sci 11:540-552.

Parker Kerrigan BC, Hossain A, Yamashita S, and Lang FF (2018) Stem cell therapy of gliomas. Prog Neurol Surg 32:124-151.

Pastrana E, Silva-Vargas V, and Doetsch F (2011) Eyes wide open: a critical review of sphere-formation as an assay for stem cells. Cell Stem Cell 8:486-498.

Patel M, Vogelbaum MA, Barnett GH, Jalali R, and Ahluwalia MS (2012) Molecular targeted therapy in recurrent glioblastoma: current challenges and future directions. Expert Opin Investig Drugs 21:1247-1266.

Platten M, Nollen EAA, Röhrig UF, Fallarino F, and Opitz CA (2019) Tryptophan metabolism as a common therapeutic target in cancer, neurodegeneration and beyond. Nat Rev Drug Discov 18:379-401.

Pommier Y (2013) Drugging topoisomerases: lessons and challenges. ACS Chem Biol 8:82-95.

Rafiq S, Hackett CS, and Brentjens RJ (2020) Engineering strategies to overcome the current roadblocks in CAR T cell therapy. Nat Rev Clin Oncol 17:147-167.

Rahman M, Deleyrolle L, Vedam-Mai V, Azari H, Abd-El-Barr M, and Reynolds BA (2011) The cancer stem cell hypothesis: failures and pitfalls. Neurosurgery 68:531-545, discussion 545 .

Ramjiawan RR, Griffioen AW, and Duda DG (2017) Anti-angiogenesis for cancer revisited: Is there a role for combinations with immunotherapy? Angiogenesis 20:185-204.

Rask-Andersen M, Almén MS, and Schiöth HB (2011) Trends in the exploitation of novel drug targets. Nat Rev Drug Discov 10:579-590.

Rask-Andersen M, Zhang J, Fabbro D, and Schiöth HB (2014) Advances in kinase targeting: current clinical use and clinical trials. Trends Pharmacol Sci 35:604-620.

Reardon DA, Brandes AA, Omuro A, Mulholland P, Lim M, Wick A, Baehring J, Ahluwalia MS, Roth P, Bahr O, et al. (2020) Effect of nivolumab vs bevacizumab in patients with recurrent glioblastoma: The CheckMate 143 phase 3 randomized clinical trial. JAMA Oncol 6:1003-1010.

Rinne ML, Lee EQ, Nayak L, Norden AD, Beroukhim R, Wen PY, and Reardon DA (2013) Update on bevacizumab and other angiogenesis inhibitors for brain cancer. Expert Opin Emerg Drugs 18:137-153.

Robbins PD and Morelli AE (2014) Regulation of immune responses by extracellular vesicles. Nat Rev Immunol 14:195-208.

Rojas-Sepúlveda D, Tittarelli A, Gleisner MA, Ávalos I, Pereda C, Gallegos I, González FE, López MN, Butte JM, Roa JC, et al. (2018) Tumor lysate-based vaccines: on the road to immunotherapy for gallbladder cancer. Cancer Immunol Immunother 67:1897-1910.

Sabado RL, Balan S, and Bhardwaj N (2017) Dendritic cell-based immunotherapy. Cell Res 27:74-95.

Sadelain M, Rivière I, and Riddell S (2017) Therapeutic T cell engineering. Nature 545:423-431.

Saha B, Wong CM, and Parks RJ (2014) The adenovirus genome contributes to the structural stability of the virion. Viruses 6:3563-3583.

Saier Jr MH, Reddy VS, Tamang DG, and Västermark A (2014) The transporter classification database. Nucleic Acids Res 42:D251-D258.

Salem ML and Cole DJ (2010) Dendritic cell recovery post-lymphodepletion: a potential mechanism for anti-cancer adoptive $\mathrm{T}$ cell therapy and vaccination. Cancer Immunol Immunother 59:341-353.

Sampson JH, Gunn MD, Fecci PE, and Ashley DM (2020) Brain immunology and immunotherapy in brain tumours. Nat Rev Cancer 20:12-25.

Sanson M, Marie Y, Paris S, Idbaih A, Laffaire J, Ducray F, El Hallani S, Boisselier B, Mokhtari K, Hoang-Xuan K, et al. (2009) Isocitrate dehydrogenase 1 codon 132 mutation is an important prognostic biomarker in gliomas. $J$ Clin Onco 27:4150-4154.

Santos R, Ursu O, Gaulton A, Bento AP, Donadi RS, Bologa CG, Karlsson A, AlLazikani B, Hersey A, Oprea TI, et al. (2017) A comprehensive map of molecular drug targets. Nat Rev Drug Discov 16:19-34.

Scherrmann JM (2002) Drug delivery to brain via the blood-brain barrier. Vascul Pharmacol 38:349-354. 
Sebestyen Z, Prinz I, Déchanet-Merville J, Silva-Santos B, and Kuball J (2020) Translating gammadelta $(\gamma \delta) \mathrm{T}$ cells and their receptors into cancer cell therapies. Nat Rev Drug Discov 19:169-184.

Sharpe AH and Pauken KE (2018) The diverse functions of the PD1 inhibitory pathway. Nat Rev Immunol 18:153-167.

Shimamoto G, Gegg C, Boone T, and Quéva C (2012) Peptibodies: a flexible alternative format to antibodies. MAbs 4:586-591.

Singleton WGB, Bienemann AS, Woolley M, Johnson D, Lewis O, Wyatt MJ, Damment SJP, Boulter LJ, Killick-Cole CL, Asby DJ, et al. (2018) The distribution, clearance, and brainstem toxicity of panobinostat administered by convection-enhanced delivery. J Neurosurg Pediatr 22:288-296.

Sperinde G, Montgomery D, and Mytych DT (2020) Clinical immunogenicity risk assessment for a fusion protein. AAPS J 22:64.

Stathis A and Bertoni F (2018) BET proteins as targets for anticancer treatment. Cancer Discov 8:24-36.

Strohl WR (2015) Fusion proteins for half-life extension of biologics as a strategy to make biobetters. BioDrugs 29:215-239.

Stroncek DF, Reddy O, Highfill S, and Panch SR (2019) Advances in T-cell immunotherapies. Hematol Oncol Clin North Am 33:825-837.

Sun Y, Bailey CP, Sadighi Z, Zaky W, and Chandra J (2020) Pediatric high-grade glioma: aberrant epigenetics and kinase signaling define emerging therapeutic opportunities. J Neurooncol 150:17-26.

Syed YY (2019) Selinexor: first global approval. Drugs 79:1485-1494.

Takebe N, Miele L, Harris PJ, Jeong W, Bando H, Kahn M, Yang SX, and Ivy SP (2015) Targeting Notch, Hedgehog, and Wnt pathways in cancer stem cells: clinical update. Nat Rev Clin Oncol 12:445-464.

Tambuyzer E, Vandendriessche B, Austin CP, Brooks PJ, Larsson K, Miller Needleman KI, Valentine J, Davies K, Groft SC, Preti R, et al. (2020) Therapies for rare diseases: therapeutic modalities, progress and challenges ahead. Nat Rev Drug Discov 19:93-111.

Tang B, Guo ZS, Bartlett DL, Yan DZ, Schane CP, Thomas DL, Liu J, McFadden G, Shisler JL, and Roy EJ (2020) Synergistic combination of oncolytic virotherapy and immunotherapy for glioma. Clin Cancer Res 26:2216-2230.

Tangutoori S, Baldwin P, and Sridhar S (2015) PARP inhibitors: a new era of targeted therapy. Maturitas 81:5-9.

Tarasov VV, Svistunov AA, Chubarev VN, Dostdar SA, Sokolov AV, Brzecka A, Sukocheva O, Neganova ME, Klochkov SG, Somasundaram SG, et al. (2019a) Extracellular vesicles in cancer nanomedicine. Semin Cancer Biol 69:212-225.

Tarasov VV, Svistunov AA, Chubarev VN, Zatsepilova TA, Preferanskaya NG, Stepanova OI, Sokolov AV, Dostdar SA, Minyaeva NN, Neganova ME, et al. (2019b) Feasibility of targeting glioblastoma stem cells: from concept to clinica trials. Curr Top Med Chem 19:2974-2984.

Terabe M and Berzofsky JA (2018) Tissue-specific roles of NKT cells in tumor immunity. Front Immunol 9:1838.

Thomas FC, Taskar K, Rudraraju V, Goda S, Thorsheim HR, Gaasch JA, Mittapalli RK, Palmieri D, Steeg PS, Lockman PR, et al. (2009) Uptake of ANG1005, a novel paclitaxel derivative, through the blood-brain barrier into brain and experimental brain metastases of breast cancer. Pharm Res 26:2486-2494.

Thurber GM, Schmidt MM, and Wittrup KD (2008) Antibody tumor penetration: transport opposed by systemic and antigen-mediated clearance. Adv Drug Deliv Rev 60:1421-1434.
Turtle CJ, Hanafi LA, Berger C, Gooley TA, Cherian S, Hudecek M, Sommermeyer D, Melville K, Pender B, Budiarto TM, et al. (2016) CD19 CAR-T cells of defined CD4+:CD8+ composition in adult B cell ALL patients. $J$ Clin Invest 126:2123-2138

Twumasi-Boateng K, Pettigrew JL, Kwok YYE, Bell JC, and Nelson BH (2018 Oncolytic viruses as engineering platforms for combination immunotherapy. Nat Rev Cancer 18:419-432.

Van Coillie S, Wiernicki B, and Xu J (2020) Molecular and cellular functions of CTLA-4. Adv Exp Med Biol 1248:7-32.

Venepalli NK, Emmadi R, Danciu OC, Chowdhery R, Cabay RJ, Gaitonde S, Aardsma N, Kothari R, Liu LC, Fischer JH, et al. (2019) Phase I study of IGFmethotrexate conjugate in the treatment of advanced tumors expressing IGF-1R. Am J Clin Oncol 42:862-869.

Verza FA, Das U, Fachin AL, Dimmock JR, and Marins M (2020) Roles of histone deacetylases and inhibitors in anticancer therapy. Cancers (Basel) 12:1664.

Vhora I, Patil S, Bhatt P, and Misra A (2015) Protein- and peptide-drug conjugates: an emerging drug delivery technology. Adv Protein Chem Struct Biol 98:1-55.

Vivier D, Sharma SK, and Zeglis BM (2018) Understanding the in vivo fate of radioimmunoconjugates for nuclear imaging. J Labelled Comp Radiopharm 61:672-692.

Wade M, Li YC, and Wahl GM (2013) MDM2, MDMX and p53 in oncogenesis and cancer therapy. Nat Rev Cancer 13:83-96.

Wang W, Jiang J, and Wu C (2020) CAR-NK for tumor immunotherapy: clinical transformation and future prospects. Cancer Lett 472:175-180.

Watanabe D and Goshima F (2018) Oncolytic Virotherapy by HSV. Adv Exp Med Biol 1045:63-84.

Weller M, Butowski N, Tran DD, Recht LD, Lim M, Hirte H, Ashby L, Mechtler L, Goldlust SA, Iwamoto F, et al.; ACT IV trial investigators (2017) Rindopepimut with temozolomide for patients with newly diagnosed, EGFRvIII-expressing glioblastoma (ACT IV): a randomised, double-blind, international phase 3 trial. Lancet Oncol 18:1373-1385.

Weller M and Le Rhun E (2020) How did lomustine become standard of care in recurrent glioblastoma? Cancer Treat Rev 87:102029.

Yamada T, Das Gupta TK, and Beattie CW (2013) p28, an anionic cell-penetrating peptide, increases the activity of wild type and mutated p53 without altering its conformation. Mol Pharm 10:3375-3383.

Yap TA, Omlin A, and de Bono JS (2013) Development of therapeutic combinations targeting major cancer signaling pathways. J Clin Oncol 31:1592-1605.

Yildirim MA, Goh KI, Cusick ME, Barabási AL, and Vidal M (2007) Drug-target network. Nat Biotechnol 25:1119-1126.

Yu JX, Upadhaya S, Tatake R, Barkalow F, and Hubbard-Lucey VM (2020) Cancer cell therapies: the clinical trial landscape. Nat Rev Drug Discov 19:583-584.

Zhan Y, Lew AM, and Chopin M (2019) The pleiotropic effects of the GM-CSF rheostat on myeloid cell differentiation and function: more than a numbers game. Front Immunol 10:2679.

Zhu G and Chen X (2018) Aptamer-based targeted therapy. Adv Drug Deliv Rev 134:65-78.

Zitron IM, Thakur A, Norkina O, Barger GR, Lum LG, and Mittal S (2013) Targeting and killing of glioblastoma with activated $\mathrm{T}$ cells armed with bispecific antibodies. BMC Cancer 13:83. 